\title{
INVESTIGATING THE EFFECT OF AN UPSTREAM SPHEROID ON TANDEM HYDROFOILS
}

\author{
A Thesis \\ presented to \\ the Faculty of California Polytechnic State University, \\ San Luis Obispo
}

\author{
In Partial Fulfillment \\ of the Requirements for the Degree \\ Master of Science in Aerospace Engineering
}

by

Joel Tynan Guerra

December 2018 
(C) 2018

Joel Tynan Guerra

ALL RIGHTS RESERVED 


\section{COMMITTEE MEMBERSHIP}

TITLE: Investigating the Effect of an Upstream

Spheroid on Tandem Hydrofoils

AUTHOR: Joel Tynan Guerra

DATE SUBMITTED: December 2018

COMMITTEE CHAIR: Graham Doig, Ph.D.

Assistant Professor of Aerospace Engineering

COMMITTEE MEMBER: David Marshall, Ph.D.

Professor of Aerospace Engineering, Department Chair

COMMITTEE MEMBER: Kira Abercromby, Ph.D.

Associate Professor of Aerospace Engineering

COMMITTEE MEMBER: Frank Fish, Ph.D.

Professor of Biology, West Chester University 


\begin{abstract}
Investigating the Effect of an Upstream Spheroid on Tandem Hydrofoils

Joel Tynan Guerra
\end{abstract}

This thesis documents a series of three dimensional unsteady Reynolds Averaged Navier-Stokes CFD simulations used to investigate the influence of an upstream prolate spheroid body on tandem pitching hydrofoils. The model is validated by performing separate CFD simulations on the body and pitching hydrofoils and comparing results to existing experimental data. The simulations were run for a range of Strouhal numbers $(0.2-0.5)$ and phase differences $(0-\pi)$. Results were compared to identical simulations without an upstream body to determine how the body affects thrust generation and the unsteady flow field.

The combined time-averaged thrust increases with Strouhal number, and is highest when the foils pitch out of phase with each other. At intermediate phase differences between $\phi=0$ and $\phi=\pi$ the leading foil produces significantly more thrust than the trailing foil, peaking at $\phi=\pi / 2$. For $S t=0.5$ this difference is $21.7 \%$.

Results indicate that adding an upstream prolate spheroid body does not significantly alter thrust results, though it does provide a small (nearly negligible) boost. Vorticity from the body is pulled downstream from the pitching foils, which interacts with the vortex generation when the vortex being generated is of the same sign as the body vorticity. This body vorticity does not affect the vorticity magnitude of the downstream vortex pairs. 


\section{ACKNOWLEDGMENTS}

First and most importantly, I would like to thank my advisor, Dr. Graham Doig, for giving me the opportunity to pursue graduate education at Cal Poly. Without this opportunity I would most likely never have stepped inside a wind tunnel lab, and I definitely would not have discovered my passion for "Colors for Doig". I have immensely enjoyed working with him the last two years. I am lucky to have been his graduate student.

I would also like to thank my committee members for their patience and time. The feedback I received from Dr. Paulo Iscold and Dr. David Marshall during my proposal was critical in helping me reframe my research. Thank you to Dr. Frank Fish, who was willing to sit on my committee all the way from Pennsylvania. Thank you to Dr. Kira Abercromby, who was happy to sit on my committee at the last minute- I'm glad you found my work interesting!

I would also like to acknowledge Dr. Jack Leifer and Dr. Mahbub Uddin. Their continued mentorship and advising long after I graduated from Trinity constantly reminds me of my phenomenal undergraduate experience. I must also express my gratitude for Mr. Colin Lang at Alamo Heights, who literally ignited my interest in rocket science, setting me on this path.

I would like to thank my fellow graduate students and friends Brandon Baldovin and Shaun Wixted. My fondest memories of Cal Poly will be of our late nights in the lofts and Kona lunches. I can't wait to see what you guys do in the future.

I would like to thank all the SLOBS from 2016-2018. Ultimate frisbee was a welcome break from my studies. Winning 2017 developmental regionals will stand as one of my greatest achievements. Thanks in particular to my fellow Texan and friend Jack Combs for always being willing to talk about our love for Whataburger, Buccees, and Shiner. 
I would like to thank my friend Eric Booker, without whom I surely would not have made it this far. Weekend trips to LA kept me sane during my first year away from home, and it was comforting knowing I had such a close friend nearby. You've got a couch with your name on it in Raleigh.

I would like to thank my parents for their continued support in my seemingly endless pursuit of higher education. I promise you I will get a job one day.

I would like to thank Lavanya Hospeti for being my confidant, soundboard, and best friend, even from 1,543 miles away. The cookies and care packages always helped, too.

Lastly, I would like to thank Blake Duckers for persuading me to take rocketry in high school. This thesis is dedicated to you. 


\section{TABLE OF CONTENTS}

$\begin{aligned} \text { Page } & \end{aligned}$

LIST OF TABLES . . . . . . . . . . . . . . . . . $\quad$ ix

LIST OF FIGURES . . . . . . . . . . . . . . . . . . . . . CHAPTER

1 Introduction . . . . . . . . . . . . . . . . . 1

1.1 Purpose of Research . . . . . . . . . . . . . . . . . 2

1.2 Literature Review . . . . . . . . . . . . . . . . . . . 5

1.2.1 Spheroids .................... 5

1.2 .2 Pitching Foils . . . . . . . . . . . . . . . 7

1.2.3 Foils with upstream bodies . . . . . . . . . . . . . . . . 10

1.2.4 Computational Methods . . . . . . . . . . . . 12

2 Computational Fluid Dynamics . . . . . . . . . . . . . . . . . 14

2.1 Governing Equations . . . . . . . . . . . . . . . . 14

2.2 Numerical Procedure . . . . . . . . . . . . . . . . . . . . 15

2.2.1 Spatial Discretization . . . . . . . . . . . 16

2.2.2 Temporal Discretization . . . . . . . . . . . . 16

2.2.3 Flow Solver . . . . . . . . . . . . . . . . . . . . 17

2.3 Turbulence Modeling . . . . . . . . . . . . . . . . . . . . . . 17

2.3.1 Spalart-Allmaras ................. 18

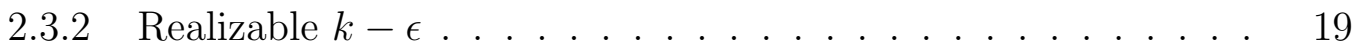

2.3.3 $k-\omega$ Shear Stress Transport . . . . . . . . . . . . . . . . . 19

2.4 Summary of Chosen Settings . . . . . . . . . . . . . . . 20

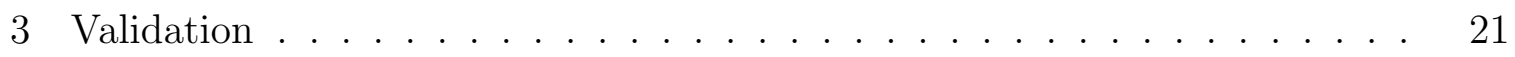

3.1 Prolate Spheroid Validation . . . . . . . . . . . . . . 21

3.1 .1 Model Setup . . . . . . . . . . . . . . . . . . . . 21

3.1.2 Grid Convergence Study . . . . . . . . . . . . . . . 24

3.1.3 Spheroid Validation Results . . . . . . . . . . . 26

3.2 Hydrofoils Validation . . . . . . . . . . . . . . . . . . 30

3.2.1 Model Setup . . . . . . . . . . . . . . . . . 30 
3.2.2 Hydrofoil Validation Results . . . . . . . . . . . . . . . . . . . 32

4 Final Simulation Setup and Results . . . . . . . . . . . . . . . . . 35

4.1 Setup . . . . . . . . . . . . . . . . . 35

4.1 .1 Model Geometry . . . . . . . . . . . . . . . 35

4.1.2 Physics Settings . . . . . . . . . . . . . . . 35

4.2 Results . . . . . . . . . . . . . . . . . . 38

4.3 Comparison to no body results . . . . . . . . . . . . . . . 47

5 Future Work . . . . . . . . . . . . . . . . . . 50

6 Conclusion . . . . . . . . . . . . . . . . . . . . . . . 52

BIBLIOGRAPHY . . . . . . . . . . . . . . . . . 54

APPENDICES 


\section{LIST OF TABLES}

Table

3.1 Domain Sizing Study . . . . . . . . . . . . . 22

3.2 Error and GCI results . . . . . . . . . . . . 26 


\section{LIST OF FIGURES}

Figure

Page

1.1 Forces generated by a swimming seal [11] . . . . . . . . . . . 2

1.2 A leopard seal. Obtained from [13]. . . . . . . . . . . . 4

1.3 Final CAD of model used in simulations. . . . . . . . . . . . . . 4

1.4 Surface skin friction and vorticity contours of a $6: 1$ prolate spheroid at $20^{\circ}$ and $R e=4.2 \times 10^{6}$. Obtained from [19]. . . . . . . . . . 6

1.5 Kinematics of a flexible pitching panel at several points in a period. The leading edge fairing does not move [23]. . . . . . . . . . . . . 8

1.6 Tandem hydrofoils in an in-line configuration [25] . . . . . . . . . 9

1.7 Tandem hydrofoils in a side-by-side configuration [17] . . . . . . . 10

1.8 A conceptual sketch of the experimental set up from Gopalkrishnan et al $[26] . \ldots \ldots \ldots \ldots 1$. . . . . . . . . . . . . . . . . . . . . 11

3.1 Spheroid validation domain. . . . . . . . . . . . . . 21

3.2 Spheroid validation cross sectional area. . . . . . . . . . . . 22

3.3 Spheroid mesh. . . . . . . . . . . . . . . . 23

3.4 Spheroid cross-sectional mesh. . . . . . . . . . . . . . . . 24

3.5 Lift and drag values with increased mesh density. . . . . . . . . . 25

3.6 Schematic showing where pressure coefficient data was taken. . . . 27

$3.7 \quad$ Coefficient of pressure comparison at $x / L=0.77$. . . . . . . . . . 28

3.8 Comparison of skin friction and vorticity results. On the left is results from Scott and Duque [16] and on the right are the results from this research. . . . . . . . . . . . . . . . . 29

3.9 Experimental separation line topology for $\alpha=20.2^{\circ}[14] \ldots$. . . . 29

3.10 Pitching fin validation domain. . . . . . . . . . . . . 30

3.11 Hydrofoils mesh, with overset regions shown in yellow. . . . . . . . 31

3.12 Coefficient of thrust versus phase differential $\phi$ for $D^{*}=0.5$. . . . 33

3.13 Comparison of experimental results to computational results for $\phi=$ $0[17] . \ldots \ldots \ldots \ldots \ldots \ldots . \ldots \ldots$

3.14 Comparison of experimental results to computational results for $\phi=$ $\pi[17] \ldots \ldots \ldots \ldots$. . . . . . . . . . . . . . . . . . . . . . . 
3.15 Comparison of experimental results to computational results for $\phi=$ $\pi / 2[17] \ldots \ldots \ldots \ldots \ldots \ldots \ldots \ldots$

$4.1 \quad$ Final model geometry. . . . . . . . . . . . . . . . . . 36

$4.2 \quad$ Final domain dimensions. . . . . . . . . . . . . . . . . 36

4.3 Final mesh, with overset meshes shown in yellow. . . . . . . . . 37

$4.4 \quad$ Pitching sequence and thrust trend for $\phi=0$ and $S t=0.5 \ldots \ldots 39$

$4.5 \quad$ Shedding events for $\phi=0$ at $S t=0.5 \ldots \ldots \ldots \ldots \ldots$

$4.6 \quad$ Pitching sequence and thrust trend for $\phi=\pi$ and $S t=0.5 \ldots \ldots 40$

$4.7 \quad$ Shedding events for $\phi=\pi$ at $S t=0.5 \ldots \ldots \ldots \ldots \ldots$

$4.8 \quad$ Pitching sequence and thrust trend for $\phi=\pi / 2$ and $S t=0.5$. . . 41

4.9 Comparison of shedding event for intermediate phase differences. The leading foil is the bottom foil. . . . . . . . . . . . . 42

4.10 Trailing foil thrust trend for increasing $\phi, S t=0.5 \ldots \ldots \ldots$

4.11 Leading foil thrust trend for increasing $\phi, S t=0.5 \ldots \ldots 44$

4.12 Combined time averaged thrust results as a function of phase difference $\phi \ldots \ldots \ldots \ldots \ldots \ldots \ldots \ldots \ldots$

4.13 Leading and trailing foil time averaged thrust results as a function of phase difference $\phi$. The dotted line is the trailing foil and the dashed line is the leading foil. . . . . . . . . . . . . . . 45

4.14 Differences in vortices and wake for different Strouhal numbers at $\phi=\pi$ and $f t=0.25 \ldots \ldots \ldots \ldots \ldots \ldots \ldots$

4.15 Pressure scalar scenes for $S t=0.5$ at $f t=1 \ldots \ldots \ldots \ldots \ldots$

4.16 Comparison of vorticity scenes for $S t=0.5$ and $\phi=\pi$ when the vortex magnitude aligns with the body magnitude. . . . . . . . 48

4.17 Comparison of vorticity scenes for $S t=0.5$ and $\phi=\pi$ when the vortex magnitude does not align with the body magnitude. . . . . 49

$4.18 C_{T}$ results for body and no body simulations. . . . . . . . . 49 


\section{Chapter 1}

\section{INTRODUCTION}

Biolocomotion is a well studied topic in fluid dynamics due in part to its potential applications in underwater vehicle propulsion. The propulsive performance and efficiency of fish and marine mammals can be significantly greater than existing manmade systems, and as a result bio-inspired propulsion is viewed as a promising alternative to conventional underwater propulsive systems.

The most straightforward way to study fish and mammalian swimming is to observe live animals in controlled environments [1-6]. In an ideal scenario, data obtained from observing live animals would be of the highest quality. However, controlling animals is difficult, because they will not always swim at a constant cadence or mode, and obtaining direct force measurements is not possible. Therefore, it can be easier to treat animals as simplified propulsive models, generally as a singular undulating body [7] or a pitching hydrofoil [8].

Using simplified models to study the force generation and resulting wake of swimming animals is possible due to caudal fins and tails resembling high aspect ratio foils [9]. Aquatic animals generate thrust by the undulatory or oscillatory motions of

their propulsive appendages [10]. Oscillatory swimmers generate thrust by pitching their tails. The tail generates a lift force perpendicular to the relative flow when angled to the free stream, which can be resolved into a thrust force in the direction of motion, shown in Figure 1.1. 


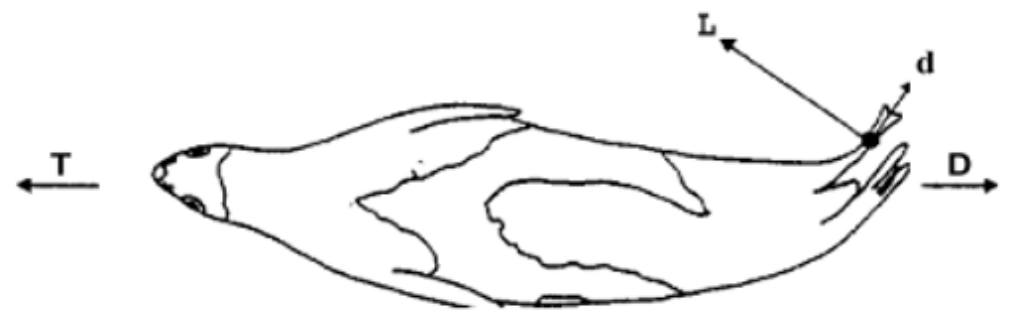

Figure 1.1: Forces generated by a swimming seal [11].

\subsection{Purpose of Research}

While using propulsive bodies to mimic fish has been successful, cetaceans and other aquatic mammals cannot be simplified as hydrofoils due to their shape and size. To investigate the propulsive performance of larger swimmers, more robust models are needed that include multiple propulsive bodies, or models with both rigid and propulsive bodies. However, work on these topics is scarce due to the added complexity.

The purpose of this work is to use computational fluid dynamics (CFD) to investigate the performance of dual pitching hydrofoils behind a rigid prolate spheroid body. This study was motivated by the lack of research on both leopard seal locomotion and on tandem fin-body interactions. The geometry is loosely based off leopard seal morphology, and the flow conditions on an average leopard seal swimming speed. An image of a typical leopard seal is shown in Figure 1.2. While the CFD model is bio-inspired, it remains significantly different from standard leopard seal morphology, detailed in [12]. The widest point of a leopard seal is its shoulders, which are found about a third of their body length down from their nose. Their flippers are crescent shaped with the ability to expand (abduction) or contract (adduction) while swimming. Seal swimming is a much more complex motion than simplified pitching foils: Not only do they use the entire posterior half to generate thrust, but they contort their flippers as well- the inner flipper contracts while the outer flipper expands. The 
fore flippers also provide a significant amount of thrust and maneuverability.

The body is approximated as a prolate spheroid, which has its maximum thickness at mid-chord. This shape was chosen due to it having an aspect ratio similar to that of a seal, and because it is a well researched shape. The hind flippers are replaced with rigid square hydrofoils. For this thesis, the results of interest were the phase difference and Strouhal number, not the influence of foil shape. In order to study the performance of the pitching hydrofoils, the fore flippers were not modeled. The entire model was also rigid, meaning the body or foils did not flex to generate additional thrust. These simplifications result in a model that should not be referred to as a seal. However, the results can still provide preliminary information about the mechanisms of leopard seal locomotion and provide an opening for more realistic future analysis. In the rest of the thesis the model will be referred to as a pair of foils with an upstream body.

The goals of this thesis are as follows:

- To conduct a series of three-dimensional unsteady Reynolds-Averaged Navier Stokes (RANS) CFD analyses of a bio-inspired model with a spheroid body upstream of tandem hydrofoils, shown in Figure 1.3.

- To investigate the effect of the upstream body by conducting a series of identical simulations without the presence of an upstream body.

- To serve as a starting point for investigation into the characteristics of seal swimming at Cal Poly by developing a moving mesh methodology that can be extended to more complex models.

Validating this work presents a challenge, since little research exists on bodies upstream of tandem foils. As a result, the simulation will be validated in two parts: The prolate spheroid will be validated to experimental results from a Virginia Tech 


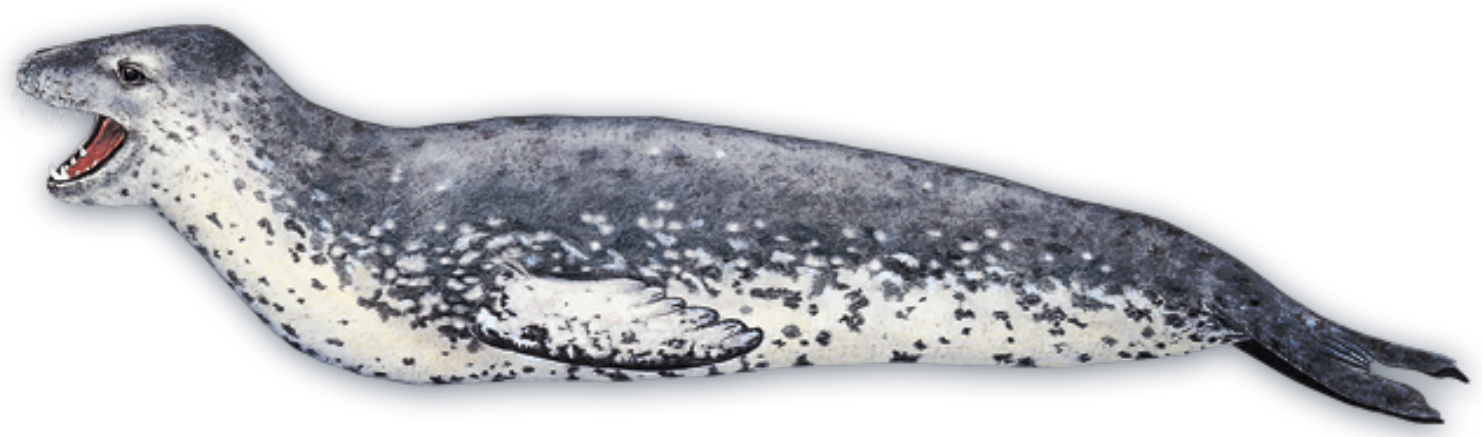

Figure 1.2: A leopard seal. Obtained from [13].

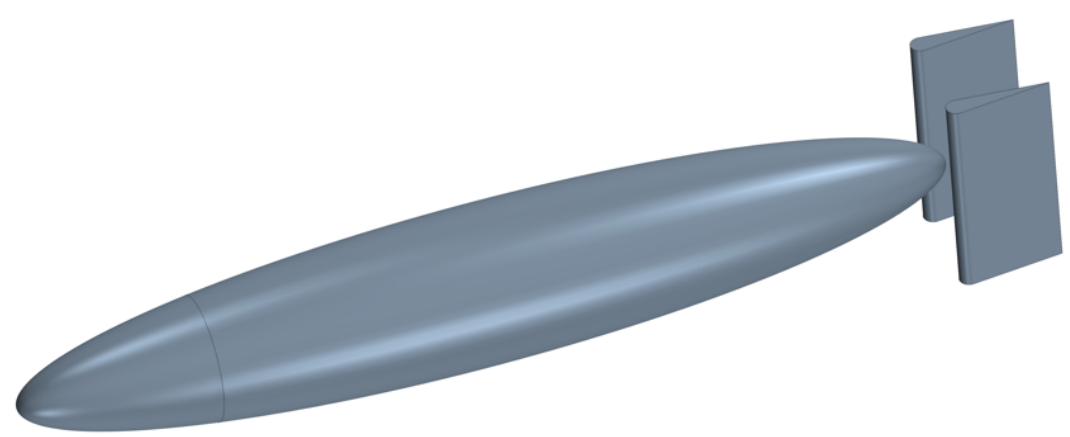

Figure 1.3: Final CAD of model used in simulations. 
campaign $[14,15]$ and numerical results on the same model from [16]. The pair of oscillating foils will be modeled identically to Dewey's experimental foils, and validated to those results [17]. With both parts validated, the geometries will be merged to form the final geometry.

\section{$1.2 \quad$ Literature Review}

This section details previous work on prolate spheroids as well as pitching hydrofoils with and without body interactions. Previous work on using CFD to investigate biolocomotion is also presented.

\subsubsection{Spheroids}

Prolate spheroids are simple shapes that produce complex flow fields, exhibiting three dimensional separation from a curved surface and regions of both laminar and turbulent flow [18]. The flow field is also qualitatively similar to that of underwater vehicles, making it a well-research shape as it can serve as a simplified case for these models [14]. The most researched size is the 6:1 prolate spheroid, corresponding to a major axis that is six times longer than the minor axis.

An extensive Virginia Tech wind tunnel campaign was run with a 6:1 prolate spheroid as the subject in the 1990's. Wetzel and Simpson studied the unsteady flow field of the model undergoing typical submarine maneuvers at a Reynolds number of 4.2 million [14]. This was the first documentation of unsteady data of the prolate spheroid, including surface pressures, skin friction, and force and moment measure-

ments [14]. In addition to the pitching maneuvers, Wetzel and Simpson also recorded steady data for a range of angles of attack and found that as the angle of attack of the spheroid increases, separation moves forward and windward and eventually splits into a forked nose separation. At angles above $15^{\circ}$ the flow can separate prior to the 


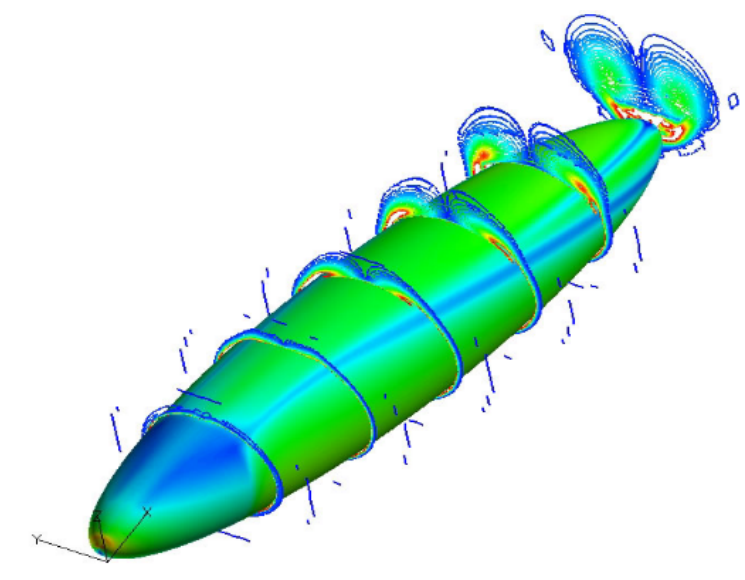

Figure 1.4: Surface skin friction and vorticity contours of a 6:1 prolate spheroid at $20^{\circ}$ and $R e=4.2 \times 10^{6}$. Obtained from [19].

trip strip located at $20 \%$ chord.

The 6:1 prolate spheroid is commonly studied using CFD because it is a simple shape that produces a flow field with complicated three-dimensional separation [14]. Accurately modeling three-dimensional separation via numerical simulations is challenging, and prolate spheroids are a simple shape to test codes on. Scott and Duque performed unsteady RANS CFD at the same conditions as [14], intending to investigate how the OVERFLOW code performed with complex separated flows [16]. Using unsteady RANS (URANS), Scott and Duque found that sufficient grid resolution in the circumferential direction is necessary to capture the pressure gradients leading to separation, and that continuity between the background and overset mesh is important. Because the $k-\omega$ SST turbulence model performed the best at modeling the $C_{P}$ distribution and capturing both separations on the leeside, the authors recommend using it for 6:1 prolate spheroids.

Figure 1.4 shows the flow field using the $k-\omega$ SST turbulence model. It should be noted that the forking separation at the nose seen in [14] is not evident here.

Rhee and Hino used a RANS method to investigate unsteady turbulent flow around a prolate spheroid pitching about its center while moving forward [20]. They 
compared steady and unsteady skin friction results at $\alpha=10^{\circ}-30^{\circ}$. The authors found that the unsteady flowfield of a spheroid at an angle of attack can have a different separation pattern than that of a steady flow field [20]. At $20^{\circ}$ the authors report that the unsteady primary separation is shifted leewards, towards the top of the spheroid. The authors also suggest that their computational approach could be applicable for more complex models, such as ones with appendages or maneuvering vehicles.

Alin et al used large eddy simulation (LES) to investigate flow around the inclined prolate spheroid at identical conditions to [14] [21]. LES is a technique that involves resolving large scale turbulence eddies while filtering out the small scale turbulence and modeling it. It is much more computationally expensive than RANS methods but can better model complex flows [21]. The authors found that the LES identified both primary and secondary separations presented in [14].

In addition to their URANS simulations, Scott and Duque conducted detached eddy simulation (DES) on a prolate spheroid model [19]. DES is a hybrid RANSLES approach that uses RANS in near-wall regions and LES everywhere else. Using RANS lowers the grid requirements near the wall, resulting in a model that is computationally cheaper than LES. Scott and Duque found that near the body results did not improve by switching from RANS to DES, which was expected since DES uses RANS near walls [19]. Switching from RANS to DES did improve the velocity profiles away from the body. Because DES completely resolves the large scales, it captures the downstream vortex street better than RANS methods.

\subsubsection{Pitching Foils}

Recently, the Princeton Gas Dynamics Lab conducted a campaign on pitching hydrofoils. For single propulsive bodies they looked at a pitching hydrofoil in ground 
effect [22]. Dewey notes that by utilizing the method of images, an unsteady propulsor in ground effect is identical to two unsteady propulsors oscillating out of phase [17]. Quinn et al found that the time- averaged thrust increased as the foil moved closer to the ground with no change in propulsive efficiency [22].

Dewey et al also investigated the performance of flexible pitching panels compared to rigid ones [23]. The experiments were conducted in a water channel with a free stream velocity of $U_{\infty}=0.06 \mathrm{~m} / \mathrm{s}$, resulting in a Reynolds number of 7200 . Figure 1.5 shows the shape of a flexible panel at several points in the cycle.

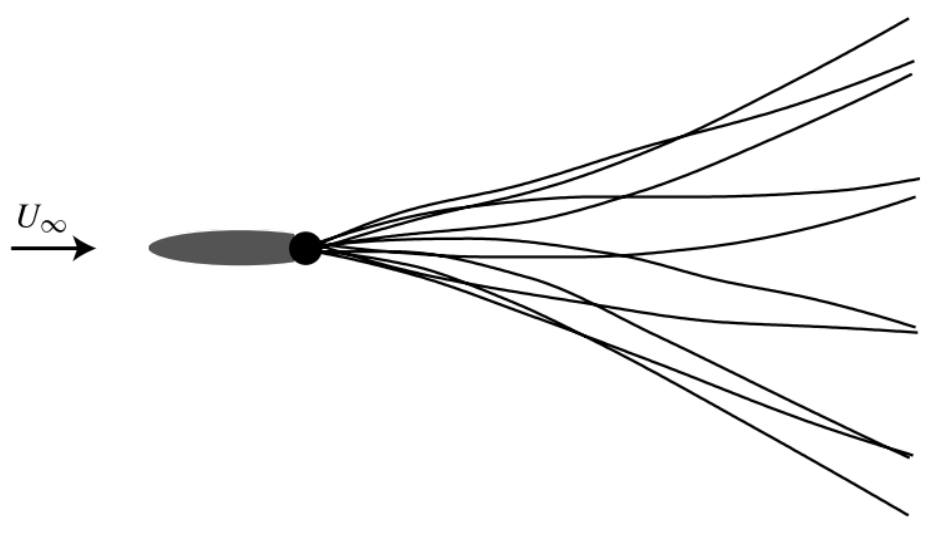

Figure 1.5: Kinematics of a flexible pitching panel at several points in a period. The leading edge fairing does not move [23].

Dewey et al found that flexible foils provide an increase in thrust production of $\mathcal{O}(100-200 \%)$ and propulsive efficiency of $\mathcal{O}(100 \%)$, which peak when the panel oscillation falls within the optimal Strouhal number range $(0.25-0.35)$ predicted by Triantafyllou et al in [24] and when the frequency is tuned to the structural resonant frequency of the panel.

Dewey et al also looked at tandem hydrofoils, investigating the thrust production and wake of both in-line and side-by-side configurations, shown in Figures 1.6 and 1.7, respectively $[17,25]$. For the in-line case, Boschitsch et al found that the upstream foil was only affected by the downstream foil for close distances, whereas the down- 
stream foil performance depended heavily on the phase difference and distance of the upstream foil [25]. The authors found two distinct wake modes, which corresponded to significant changes in the downstream foil performance. In the coherent mode, the time-averaged wake is a concentrated jet of high-momentum fluid and is associated with a peak in thrust and propulsive efficiency 1.5 times as high as an isolated foil. In the branched mode, the time-averaged wake is a branched wake that contains a pair of angled high-momentum jets and a decrease in thrust and propulsive efficiency that can be as low as 0.5 times that of an isolated foil.

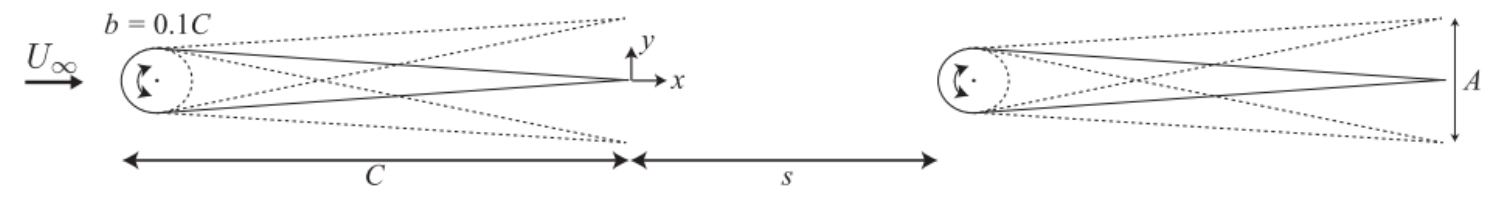

Figure 1.6: Tandem hydrofoils in an in-line configuration [25].

For the side-by-side case, the thrust production and power input to the fluid both depended on the phase difference and distance between foils. When the foils pitched in-phase, an increase in propulsive efficiency was observed at the expense of reduced thrust. When the foils pitched out of phase the thrust production increased with no significant change to propulsive efficiency. When the foils pitch with intermediate phase differences one foil sees an increase in thrust and power consumption, and the other foil sees reduced values. The wake shedding depends heavily on the phase difference. 


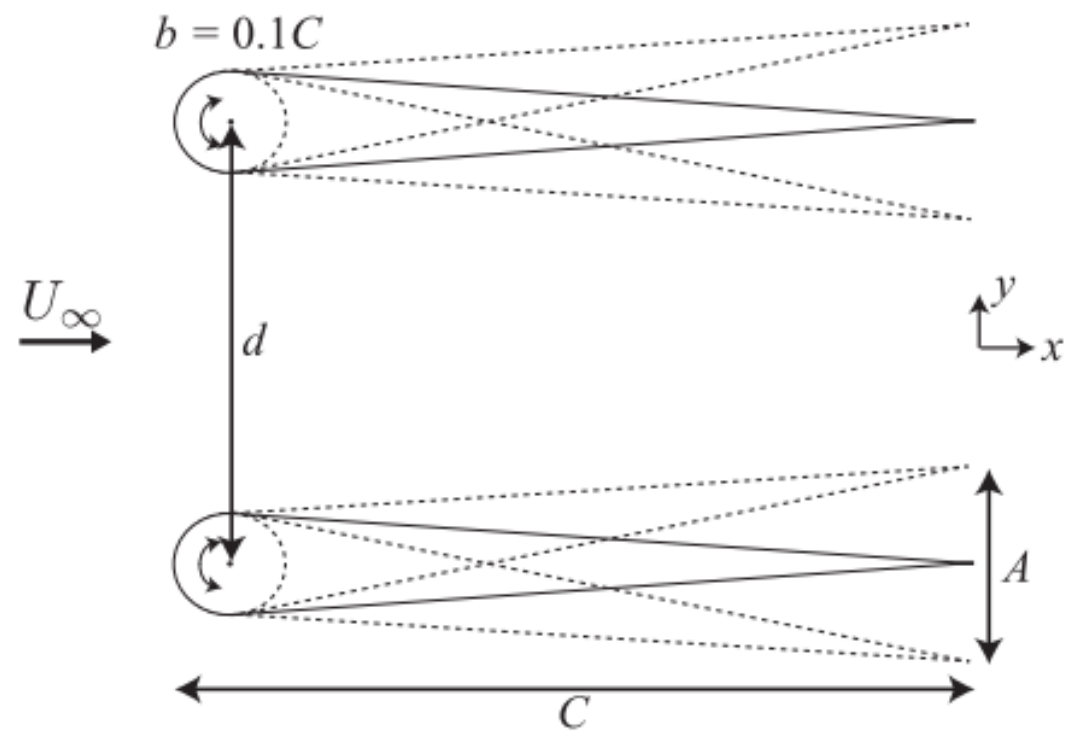

Figure 1.7: Tandem hydrofoils in a side-by-side configuration [17].

\subsubsection{Foils with upstream bodies}

Researching propulsive surfaces with upstream disturbances is more scarce. Gopalkrishnan et al investigated a flapping foil downstream of a rotating cylinder shedding coherent vortices with the goal of manipulating free shear flows through vortex control [26]. A sketch of the concept is shown in Figure 1.8. The downstream foil was pitched at a frequency near the vortex shedding frequency, and flow control was achieved by varying the foil pitching phase. The authors found that the foil efficiency depended on the phase difference between the foil pitching frequency and the frequency of the shed vortices. While the main intent of this paper was to illustrate flow control capabilities, the authors also noted that it could be applied to fish swimming. 


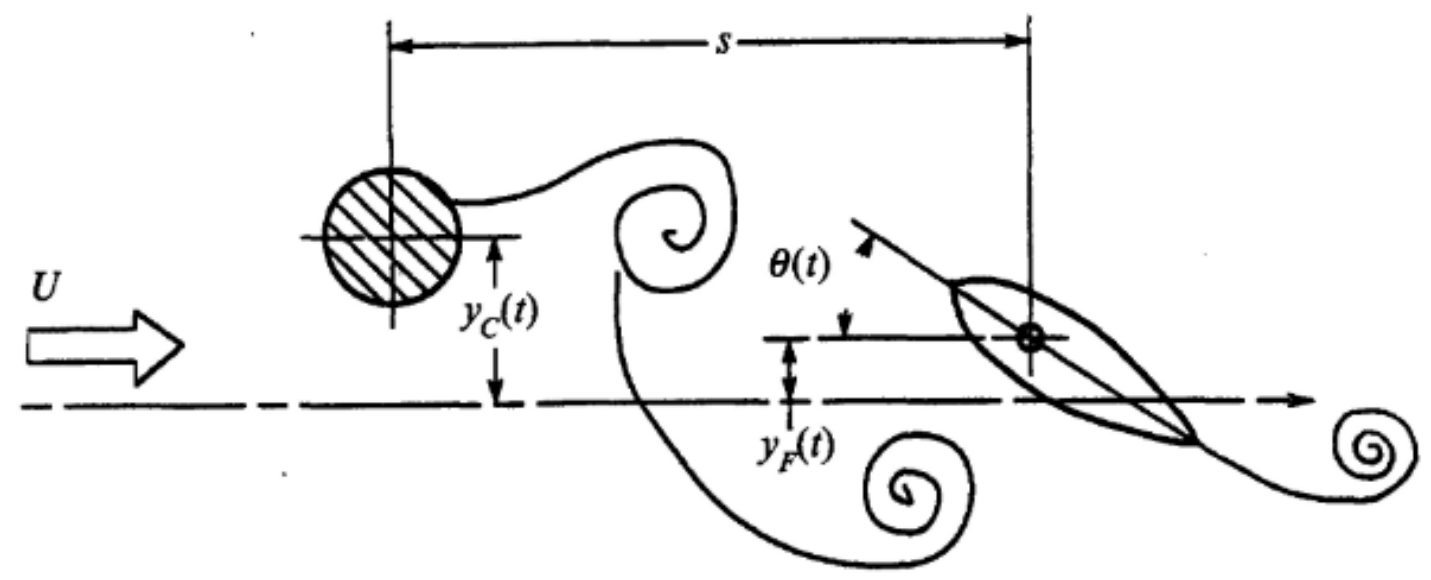

Figure 1.8: A conceptual sketch of the experimental set up from Gopalkrishnan et al [26].

Donnelly et al investigated the unsteady hydrodynamics of tandem foils behind a cylindrical body with a swaying head, meant to mimic a fish. They found that the swaying of the head is coupled with the pitching tail and produces unsteady force vectors ideal for precision maneuvers [27]. They also found the efficiency reached a peak below the Strouhal number (St) range of $0.25-0.35$ predicted in [24].

Triantafyllou documents experimental research on biomimetic foils leading up to 2004 in [9]. The majority of the work discussed is on two- and three-dimensional steady pitching foils, but there are sections detailing research on multiple foils, foilbody interactions, and foil interactions with upstream unsteady flows. A reduction in performance can occur when the wakes of dual pitching foils interact. For optimal performance the foils need to be sufficiently separated such that the wakes of the foils do not destructively interfere. Triantafyllou states that studying the wake of an upstream body and downstream foil requires understanding of the interactions between externally generated vorticity and foil generated vorticity, and how it affects propulsive performance. 


\subsubsection{Computational Methods}

Decades of advances in computing power have resulted in the continuous improvement of computational fluid dynamics for dynamic models. Simulating a swimming fish requires significant computing power to properly model the complexities of the flow field, which is why it was not attempted until the 1990's. Using CFD to investigate unsteady foils or undulating bodies has potential to be faster and easier than building a model and testing it, and can be used to investigate more complex problems than would be practical for an experimental set up.

Liu et al claim to have conducted the first CFD simulation of unsteady 3D undulatory locomotion, modeling a swimming tadpole [7] at a Reynolds number of 7200 . They compared results to a 2D study from the previous year [28]. Flanagan's Masters research was on CFD of two dimensional rainbow trout swimming, a well-researched motion and geometry [29]. The computational results agree well with experimental results. He reports that the boundary layer is laminar and the trout model has a lower than expected efficiency of $62 \%$, but a reasonable power output result near the results obtained in [30].

Chang et al studied the effect of Reynolds number on an undulating tuna-like body [31]. The authors tested the Spalart-Allmaras and k- $\omega$ SST turbulence models and compared them to a laminar case. They found that using turbulence models at higher Reynolds numbers produced better propulsive performance since the flow stays attached to the body for longer. They also compared the performance of three different caudal fin shapes and found that a crescent shape is the most efficient design because it has less lateral power loss than semicircle and fan-shaped fins. This could be attributed to the smaller surface area of the crescent-shaped fin.

More recently, Borazjani and Daghooghi used CFD to study leading edge vortices of three different tail shapes attached to a mackerel body [32]. They show that near 
Strouhal numbers of 0.25 an attached leading edge vortex (LEV) is formed which provides additional thrust. At higher values near 0.6 the LEV does not reattach.

Dong et al used CFD to study fin-fin and body-fin interactions of a fish model [33]. In this study, the fin-fin interactions refer to dorsal/ventral fin interactions with the caudal fin, as opposed to two side-by-side fins interacting as in [17]. They found that the LEV generated most of the thrust and can be augmented via capturing the vortices shed off the undulating posterior body. 
Chapter 2

\section{COMPUTATIONAL FLUID DYNAMICS}

STAR-CCM+ version 12.06 was used to model the flow field by solving the unsteady three-dimensional turbulent Navier-Stokes equations. All simulations were run using 24 cores on Cal Poly's Bishop high performance computing (HPC) cluster. STAR$\mathrm{CCM}+$ was chosen due to the author's experience with the program, and available licensing through Cal Poly.

\subsection{Governing Equations}

The governing equations that are used to describe the flow behavior are derived from the conservation laws of physics. The conservation of mass law states that the rate of change of mass within a volume must equal the mass flux across the volume surface. The continuity equation, shown in differential form as 2.1, is the conservation of mass law in equation form.

$$
\frac{\partial \rho}{\partial t}+\nabla \cdot(\rho \vec{u})
$$

The flow in question can be assumed to be turbulent, unsteady, incompressible, and three-dimensional. Assuming incompressibility means there is no spatial or temporal change in density, which simplifies equation 2.1 to:

$$
\nabla \cdot \vec{u}=0
$$

Conservation of momentum, or Newton's Second Law, states that the sum of the forces acting on a body are equal to its mass times acceleration, or equivalently, the time rate of change of momentum of the body.

$$
\sum \vec{F}=m \vec{a}=\frac{D \vec{u}}{D t}
$$


The Navier-Stokes equations are Newton's Second Law applied to a moving fluid element. The three equations (for three-dimensional flows) can be written as:

$$
\rho \frac{D \vec{u}}{D t}=-\nabla \bar{p}+\mu \nabla^{2} \vec{u}+\frac{1}{3} \mu \nabla(\nabla \cdot \vec{u})+\rho \vec{g}
$$

Conservation of energy states that the rate of change of energy of a body is equal to the change of work done on the system plus the change of heat added to the system. Since incompressibility is assumed, and there is no heat transfer, the energy equation was not used.

Because the Navier-Stokes equations are non-linear, they cannot be solved by hand. However, CFD allows users to solve modified Navier-Stokes equations known as the Reynolds Averaged Navier Stokes (RANS) equations by time-averaging the equations and solving them iteratively on a discretized fluid domain.

\subsection{Numerical Procedure}

Most current CFD codes utilize the finite volume method (FVM) over the finite difference method (FDM). The FDM uses the differential form of the governing equations while the FVM uses the integral form. Using the integral form of the governing equations over the differential form allows for a few advantages: FVM handles the conservation laws better than FDM when the mesh is a finite size, and it handles complex domains easier [34].

Star-CCM+ uses the FVM approach to solve the partial differential equations. FVM requires that the fluid domain is broken into several tiny control volumes (cells) that together are called the mesh. Mesh generation is one of the most critical steps in CFD. Placing a higher volume of cells in areas of significant flow gradients will generally result in a more accurate solution, while the cells far away from the region of interest can be larger to save computing power. 


\subsubsection{Spatial Discretization}

The fluid domain is broken into a mesh so the governing equations can be discretized in space, being solved for each control volume. Information from upstream cells is passed to the downstream cells. Hence, these discretization schemes are called upwinding schemes. STAR-CCM+ offers both first- and second-order solvers, as well as a hybrid third order central differencing scheme. The first order scheme uses information at and directly behind the point of interest, which makes it economical and stable, but not very accurate. First order schemes are only recommended to initialize a solution or when a higher order scheme does not converge. Second order solvers introduce a third term via a Taylor series expansion that results in a more accurate solution than first order solvers. Because there were no convergence issues, the second order upwinding scheme was used in this study.

\subsubsection{Temporal Discretization}

For unsteady problems the transient behavior of the flow must be accounted for by discretizing the solution, into small time steps. The same approach for solving spatial derivatives can be used to solve temporal ones. However, time derivatives can only be one-sided since the future flow field is unknown [34]. The two options for time integration are explicit methods, and implicit methods. Explicit methods solve for the next time step using information only obtained from the current step. While explicit schemes are straightforward, they have long solve times since the time step needs to be very small to remain stable. Implicit schemes require the system of equations to be solved at each time step, but are more stable than explicit schemes. They can

handle complex problems faster and more efficiently than explicit schemes due to their stability. 


\subsubsection{Flow Solver}

STAR-CCM+ offers coupled and segregated flow solvers to solve the conservation equations of mass and momentum. The segregated solver solves these equations iteratively for velocity and pressure and is appropriate for constant density flows. Because it solves the governing equations iteratively there is less memory requirement and the simulation will converge rapidly. Segregated flow also requires an implicit time scheme.

The coupled solver solves the governing equations simultaneously as a vector of equations. Solving the coupled equations simultaneously requires more memory than solving them sequentially, but can yield more accurate results in less iterations. Both explicit and implicit time scheme options are available for the coupled flow solver.

The segregated flow solver was chosen since the flow is incompressible and has constant density, and because the Bishop HPC cluster was used, the increased solve time offered by the coupled solver was not needed.

\subsection{Turbulence Modeling}

The RANS equations split the Navier-Stokes equations into mean and fluctuating components. The mean components are found relatively easily, but the fluctuating components are associated with turbulence and become problematic. Equation 2.5 is the Navier-Stokes equation in the $x$-direction, and the rightmost term inside the brackets is called the Reynolds stress. This term introduces additional unknowns,

creating an issue in solving the resultant system of equations that is famously known as the closure problem.

$$
\rho u+\frac{\partial u}{\partial x}+\rho v \frac{\partial u}{\partial x}=-\frac{\partial p}{\partial x}+\frac{\partial}{\partial y}\left[\mu \frac{\partial u}{\partial y}-\rho \overline{u^{\prime} v^{\prime}}\right]
$$


There are two ways to solve for the Reynolds stress. The most direct methods are knows as Reynolds stress models (RSM), which can be quite complex and can contain seven equations, one for each stress component and dissipation term [34]. A simpler method is to relate the Reynolds stress to the mean strain rate, shown in Equation 2.6, where $\mu_{t}$ is the eddy viscosity and $k$ is the turbulent kinetic energy. This assumption is called the Boussinesq hypothesis, which most common modern turbulence models are based on.

Turbulence models are different ways of relating the Reynolds stress term to known flow values.

$$
\tau_{i j}=2 \mu_{t} S_{i j}^{*}-\frac{2}{3} \rho k \delta_{i j}
$$

\subsubsection{Spalart-Allmaras}

The Spalart-Allmaras model is a one-equation turbulence model that solves a single transport equation for the eddy viscosity. It offers good results for boundary layers with adverse pressure gradients and wall bounded flows. The turbulent eddy viscosity can be found using Equation 2.7.

$$
\mu_{t}=\rho \tilde{\nu}_{t} f_{\nu 1}
$$

where $\tilde{\nu_{t}}$ is the turbulent kinetic viscosity and $f_{\nu 1}$ is the damping function given by Equation 2.8.

$$
f_{\nu 1}=\frac{X^{3}}{X^{3}+c_{\nu 1}^{3}}
$$

where $X=\frac{\tilde{\nu}}{\nu}, c_{\nu 1}=7.1$ and $\nu$ is the molecular kinematic viscosity. The SpalartAllmaras one equation model is easier to use than early two-equation models since 
they require finer grids and involve extra terms that negatively impact the convergence $[35]$.

\subsubsection{Realizable $k-\epsilon$}

The k-epsilon $(\mathrm{k}-\epsilon)$ turbulence models are two-equation models, solving two transport equations for the turbulent kinetic energy $(\mathrm{k})$ and the rate of dissipation of turbulent energy $(\epsilon)$. They were designed for high Reynolds number turbulent flows. The realizable $\mathrm{k}-\epsilon$ model has a few improvements over the original model, namely that it includes a new formulation for the turbulent viscosity and a new transport equation for the dissipation rate. It more accurately predicts separation and rotational flows than the original model, which had weaknesses in these applications. The eddy viscosity for $\mathrm{k}-\epsilon$ models is calculated using Equation 2.9.

$$
\mu_{t}=\rho C_{\mu} \frac{k^{2}}{\epsilon}
$$

In the standard k- $\epsilon$ model, $C_{\mu}$ is a constant, however in the realizable k- $\epsilon$ model it is a function of the mean strain rate and the rotational rate [36].

\subsection{3 $k-\omega$ Shear Stress Transport}

The k- $\omega$ turbulence models are also two-equation models, which solve transport equations for the turbulent kinetic energy and the specific dissipation rate $\omega$. The baseline $\mathrm{k}-\omega$ model was considered an improvement over the $\mathrm{k}-\epsilon$ model in that it utilizes the original k- $\omega$ model in the lower boundary layer and switches to $k-\epsilon$ in the outer region and in free shear flows [37]. The shear stress transport (SST) model includes a modification to the eddy viscosity calculation which results in better prediction of adverse pressure gradients. The eddy viscosity for the k- $\omega$ SST model is calculated 
using Equation 2.10, where $a_{1}$ is a constant and $F_{2}$ is equal to one for boundary layer flows and zero for free shear flows [37].

$$
\mu_{t}=\frac{\rho a_{1} k}{\max \left(a_{1} \omega, \Omega F_{2}\right)}
$$

The k- $\omega$ SST turbulence model was ultimately chosen because the model was used successfully in [31] and was recommended in [16].

\subsection{Summary of Chosen Settings}

Unless otherwise stated, the following models and settings were used for the numerical models in the following chapters. The flow is assumed to be unsteady, constant density, incompressible, and turbulent. The $k-\omega$ SST turbulence model was used. The segregated flow solver with an upwind second order convection scheme was chosen. Choosing the segregated solver requires an implicit time scheme, and first order temporal discretization was selected. 
Chapter 3

\section{VALIDATION}

Because the final simulation model is a geometry that has never been researched, there are no existing data to validate the model. To ensure the mesh and chosen physics settings were correctly modeling the flow, the geometry was broken into two parts in order to be validated. Separately, the body and pitching foils both are well researched topics with existing experimental results to compare to.

\subsection{Prolate Spheroid Validation}

\subsubsection{Model Setup}

A 6:1 prolate spheroid was chosen as the upstream body and was meant to serve as a simplified approximation of the leopard seal body, with similar dimensions. This shape was chosen because the aspect ratio is close to the average leopard seal fineness ratio of 5.3 [12] and because 6:1 prolate spheroids are by far the most researched prolate spheroid size, making validating the computational model easy.

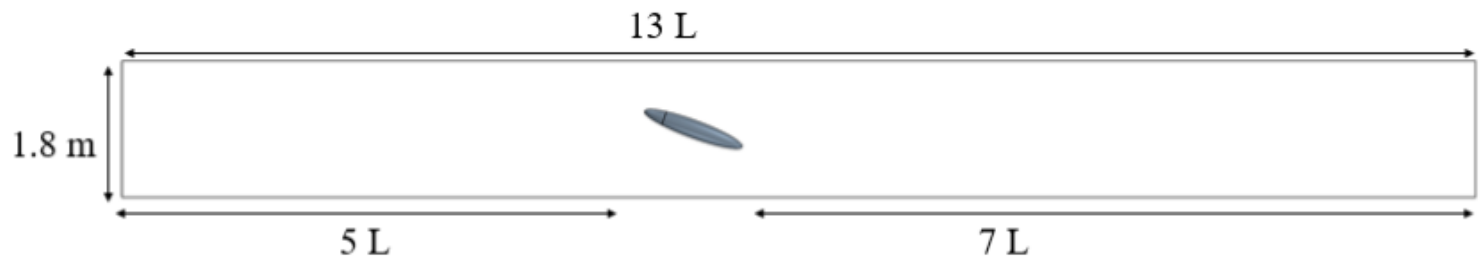

Figure 3.1: Spheroid validation domain. 


\section{Table 3.1: Domain Sizing Study}

\begin{tabular}{|l|l|l|}
\hline & Lift $(\mathrm{N})$ & $\operatorname{Drag}(\mathrm{N})$ \\
\hline $5 \mathrm{~L}$ & 14.09 & 7.29 \\
\hline $7 \mathrm{~L}$ & 13.85 & 6.92 \\
\hline $10 \mathrm{~L}$ & 13.85 & 6.92 \\
\hline
\end{tabular}

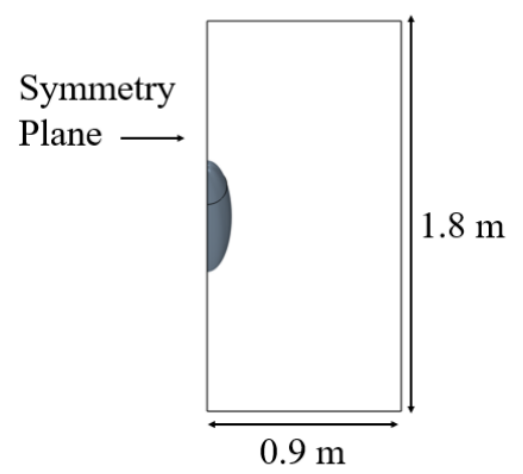

Figure 3.2: Spheroid validation cross sectional area.

The prolate spheroid simulation was compared to experimental results from a Virginia Tech wind tunnel campaign $[14,15]$ as well as computational results on the same model $[16,19]$. The simulation was set up to replicate the wind tunnel tests. The domain is shown in Figures 3.1 and 3.2, where the domain height and width are identical to the Virginia Tech test section size. A symmetry plane was used to reduce cell count. There was no mention of test section length, so a domain sizing study was done to determine how close the outlet could be, shown in Table 3.1. As a result of the domain study, the outlet was set to be 9.59 meters behind the spheroid trailing edge, or seven times the chord length.

The Reynolds number was $4.2 \times 10^{6}$, corresponding to an inlet velocity of $U_{\infty}=$ $48.02 \mathrm{~m} / \mathrm{s}$. The spheroid length is $1.37 \mathrm{~m}$, and was at a $20^{\circ}$ angle of attack. The boundary layer was artificially tripped at $x / L=0.2$ by suppressing the turbulent 
specification on the model surface ahead of the location. This was done to simulate a trip strip at the same chord length $[14,15]$. The shear stress specification for the domain walls was changed to slip so the wall boundary layer could be ignored to reduce cell count and solve time. The lift and drag values showed less than $8 \%$ difference between slip and no-slip, so this was deemed to be an acceptable simplification.

A trimmed mesh was chosen for a few reasons. Trimmed meshes are generated much faster than polyhedral meshes and result in a high accuracy since the mesh is aligned with the free stream. Since the spheroid and hydrofoils are both simple geometries, the robustness that the polyhedral mesh offers was not needed. Details of the mesh can be seen in Figures 3.3 and 3.4.

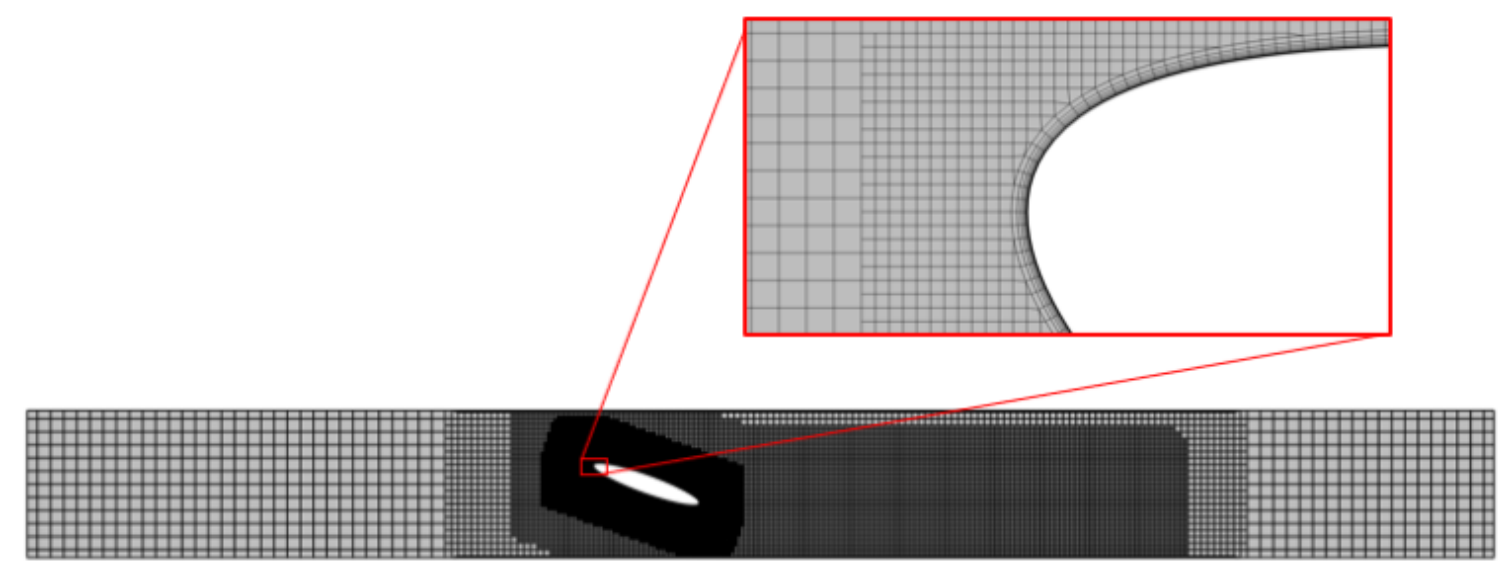

Figure 3.3: Spheroid mesh. 


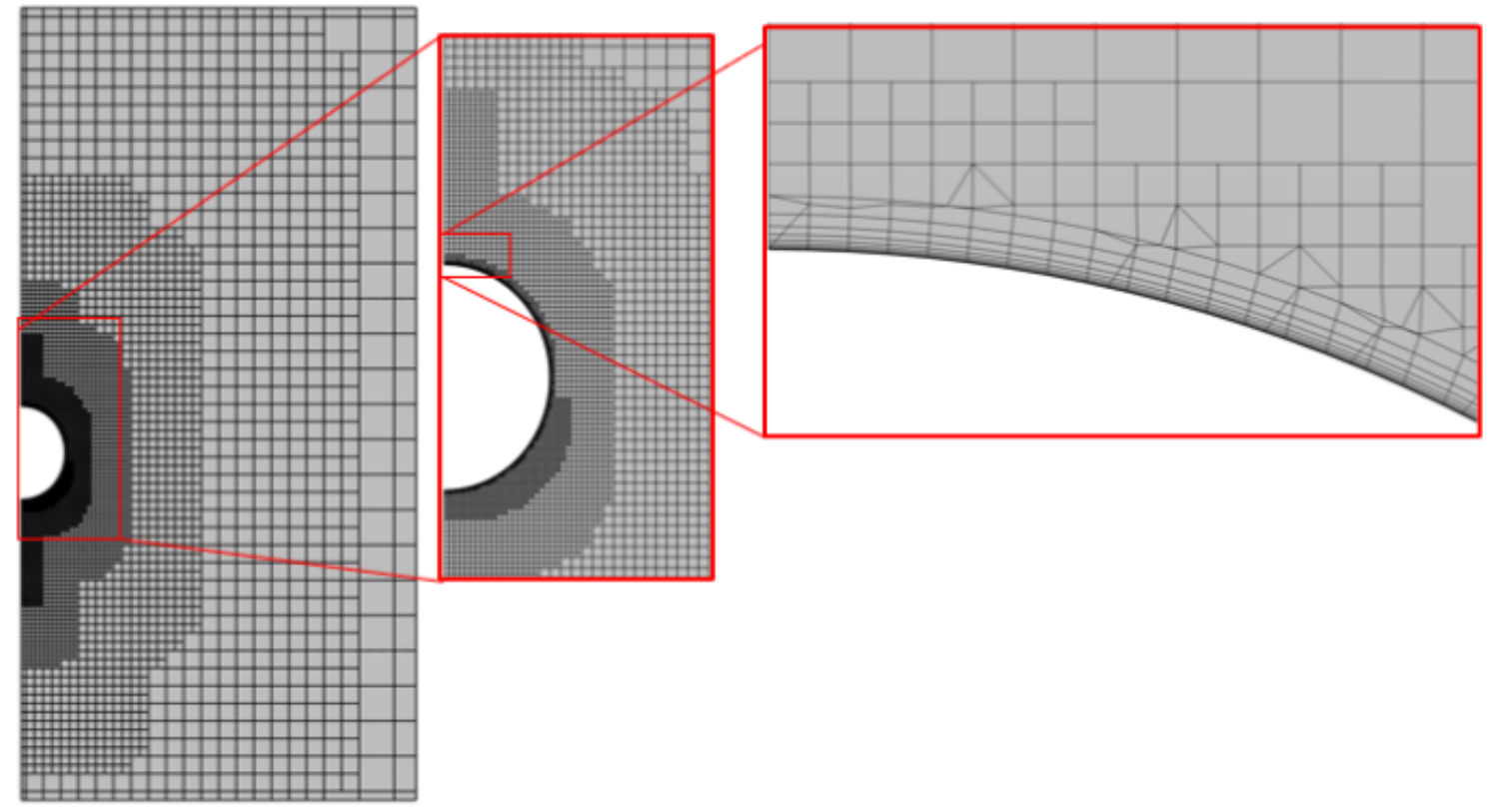

Figure 3.4: Spheroid cross-sectional mesh.

Additional refinement in the circumferential direction on the spheroid surface was needed to resolve the circumferential direction and capture the pressure gradients [16]. The wall $\mathrm{y}+$ was below 3 on the entire spheroid surface. The final spheroid mesh had a total cell count of 2.2 million cells.

\subsubsection{Grid Convergence Study}

Three meshes of coarse, medium, and fine qualities were considered. To refine the coarse mesh, the number of cells near the spheroid surface and in the wake were increased. The lift and drag values for the spheroid were used to determine grid convergence. The grid convergence index (GCI) method detailed in [38] was used to determine which mesh to use.

$$
G C I^{\text {med }}=F_{s}\left|E^{\text {med }}\right| \quad G C I^{\text {coarse }}=F_{s}\left|E^{\text {coarse }}\right|
$$




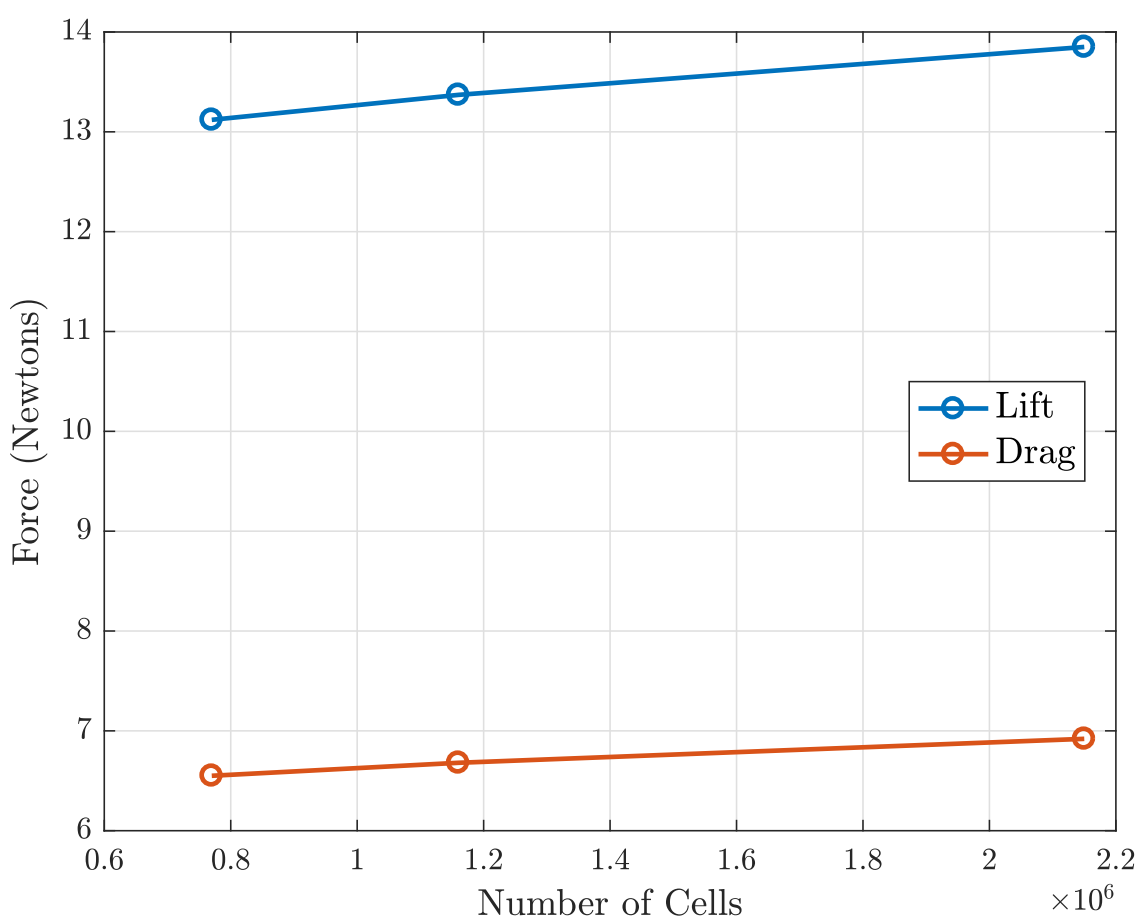

Figure 3.5: Lift and drag values with increased mesh density.

As can be seen in Figure 3.5, there is less than a four percent difference in both lift and drag values between the coarse and fine mesh. As a result, the finest mesh was ruled out and the GCI was calculated for the coarse and medium meshes. GCI is calculated using Equation 3.1.

$$
\begin{gathered}
E^{\text {med }}=\frac{\varepsilon}{\left(r^{p}-1\right)} \quad E^{\text {coarse }}=\frac{r^{p} \varepsilon}{\left(r^{p}-1\right)} \\
\varepsilon=\frac{f_{\text {coarse }}-f_{\text {medium }}}{f_{\text {coarse }}} \\
r=\frac{h_{\text {coarse }}}{h_{\text {med }}}
\end{gathered}
$$

$F_{s}$ is the factor of safety, dependent on the number of mesh intervals considered and is recommended to be 3 by Roache [38]. $E$ corresponds to the error in one 
Table 3.2: Error and GCI results

\begin{tabular}{|c|l|l|l|l|}
\cline { 2 - 5 } \multicolumn{1}{c|}{} & \multicolumn{2}{c|}{ Coarse } & \multicolumn{2}{c|}{ Medium } \\
\cline { 2 - 5 } \multicolumn{1}{c|}{} & $E$ & $G C I$ & $E$ & $G C I$ \\
\hline Lift & -0.053 & 0.159 & -0.034 & 0.102 \\
\hline Drag & -0.053 & 0.159 & -0.034 & 0.102 \\
\hline
\end{tabular}

direction and is calculated using Equation 3.2 where $\varepsilon$ is found using Equation 3.3. $h$ is the characteristic mesh length; In this case $h_{\text {coarse }}=0.02$ and $h_{\text {med }}=0.01$. Second-order accuracy is used for all solutions, meaning $p=2 . \quad f$ corresponds to the parameters under consideration, which in this case are the lift and drag on the spheroid. Results from the grid convergence study are shown in Table 3.2. Because the GCI did not change from the coarse mesh size to the medium size, the coarse base size was chosen for the mesh.

\subsubsection{Spheroid Validation Results}

Results of interest were the circumferential pressure coefficient distribution at $x / L=$ 0.77 and a scalar scene of the surface skin friction and shed vorticity.

Figure 3.7 shows the comparison between computational and experimental results for the circumferential pressure coefficient distribution, taken at $77 \%$ chord, shown in detail in Figure 3.6, where $0^{\circ}$ is the bottom of the spheroid and $180^{\circ}$ the top. The results for this were obtained by taking the coefficient of pressure on the spheroid surface at $77 \%$ chord, and averaging with the results at $76 \%$ and $78 \%$ chord. This was done to smooth the results, which looked odd in some spots due to recording scalar data on derived mesh plane. 

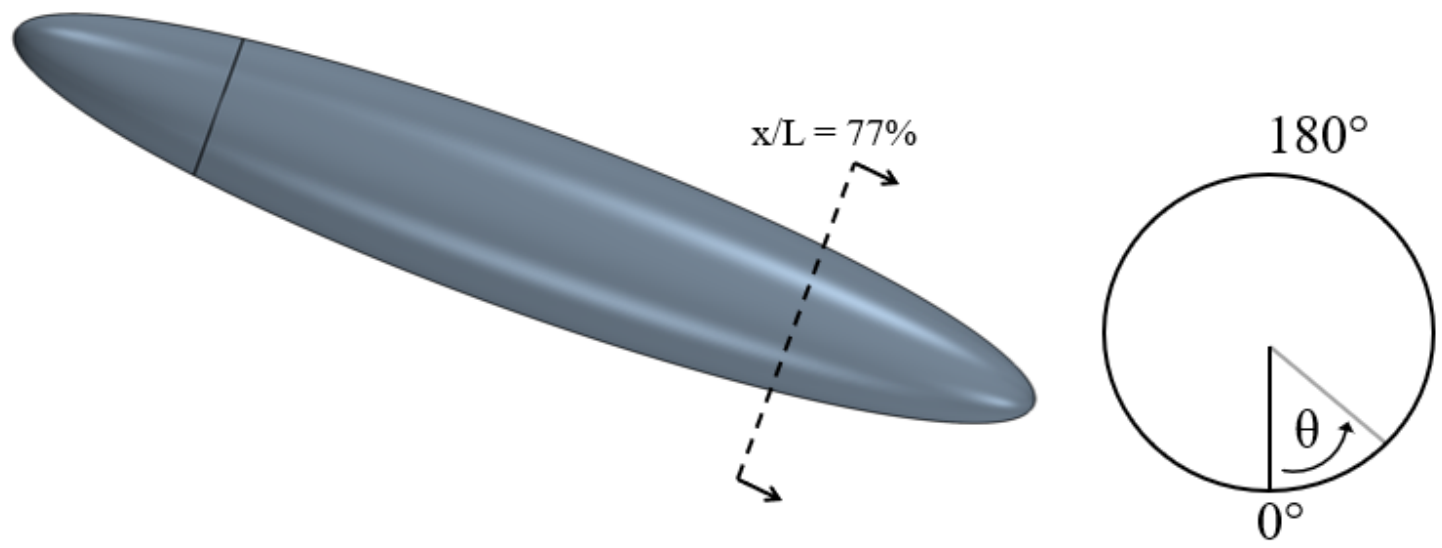

Figure 3.6: Schematic showing where pressure coefficient data was taken.

The results from this study slightly overpredicts the suction at $80^{\circ}$ and underpredicts the dip at $160^{\circ}$, however this was also seen in [16]. However, the trend is accurate. Figure 3.8 shows the comparison of the authors simulation versus Scott and Duque's results from [16]. The primary vortex is visible on the surface, shown as the streak of low skin friction stretching from the top of the spheroid to the bottom of the trailing edge. The obvious difference between the two figures is that from this study there are streaks of high skin friction stretching into the laminar zone. Here the flow is separating and transitioning before the $20 \%$ artificial trip strip. While this is different from the results published in [16], it does match up with the experimental results from Wetzel, which shows separation upstream of $20 \%$ chord, visible in Figure 3.9 [14]. The difference in computational results could be attributed to a few differences: Duque and Scott performed their simulations using a fourth order central differencing scheme with additional second and fourth order central difference dissipation terms to maintain stability [19]. The study presented in this thesis used a second order scheme. Duque and Scott also performed an unsteady simulation with first order time difference, while this study used a steady solver. Lastly, the mesh 
used in [19] had 4.89 million cells, while the mesh presented here had 2.2 million with a symmetry plane. This mesh is lower in cell count because the body in the final simulation is at $0^{\circ}$ and will not have large separation features. Scott and Duque did not mention other critical details about their setup, such as the domain size, wall $\mathrm{Y}+$, or turbulence specification. Because the CFD settings are not completely identical, there will be some differences in the solutions.

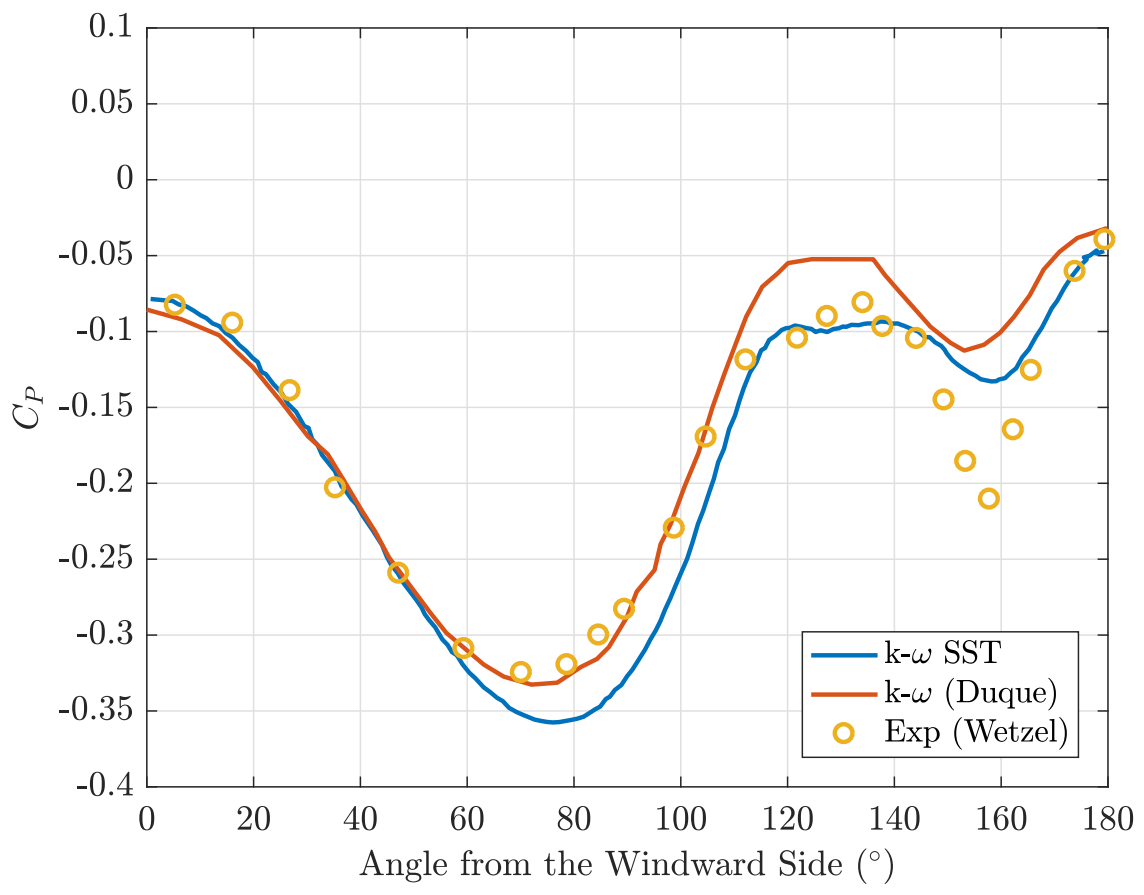

Figure 3.7: Coefficient of pressure comparison at $x / L=0.77$.

While there are some slight differences between these results and prior ones, they were encouraging enough to continue on to the pitching hydrofoils case. It is also noted that the final simulation case will have the spheroid at $0^{\circ}$ angle of attack, where there will not be any separated flow. 

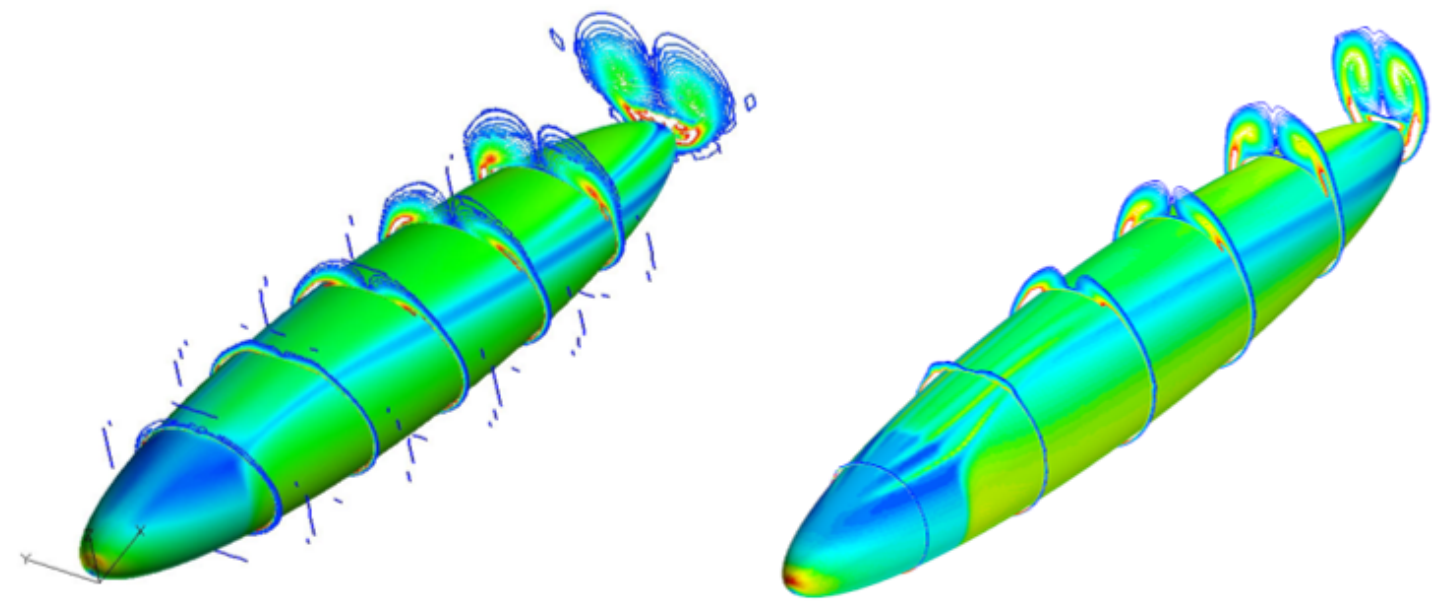

Figure 3.8: Comparison of skin friction and vorticity results. On the left is results from Scott and Duque [16] and on the right are the results from this research.

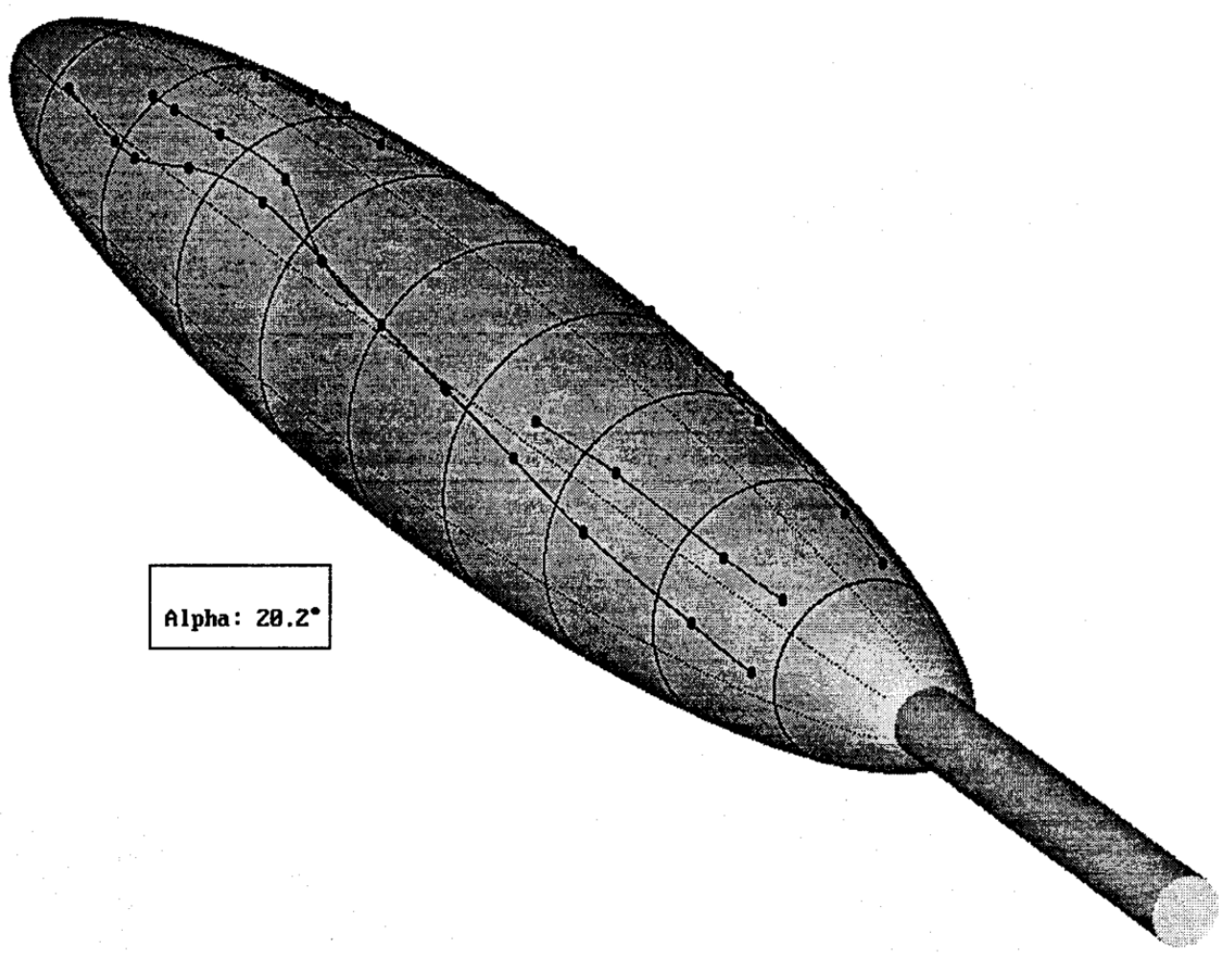

Figure 3.9: Experimental separation line topology for $\alpha=20.2^{\circ}$ [14]. 


\subsection{Hydrofoils Validation}

\subsubsection{Model Setup}

The hydrofoils were validated by comparing results to experimental data presented in [17]. As was the case with the spheroid, the experimental setup from Dewey was replicated as best as possible in STAR-CCM+, with a symmetry plane being used to reduced cell count. The streamwise position of the foils was not mentioned in the report, so the leading edges were placed one meter downstream from the inlet. The domain is shown in Figure 3.10.

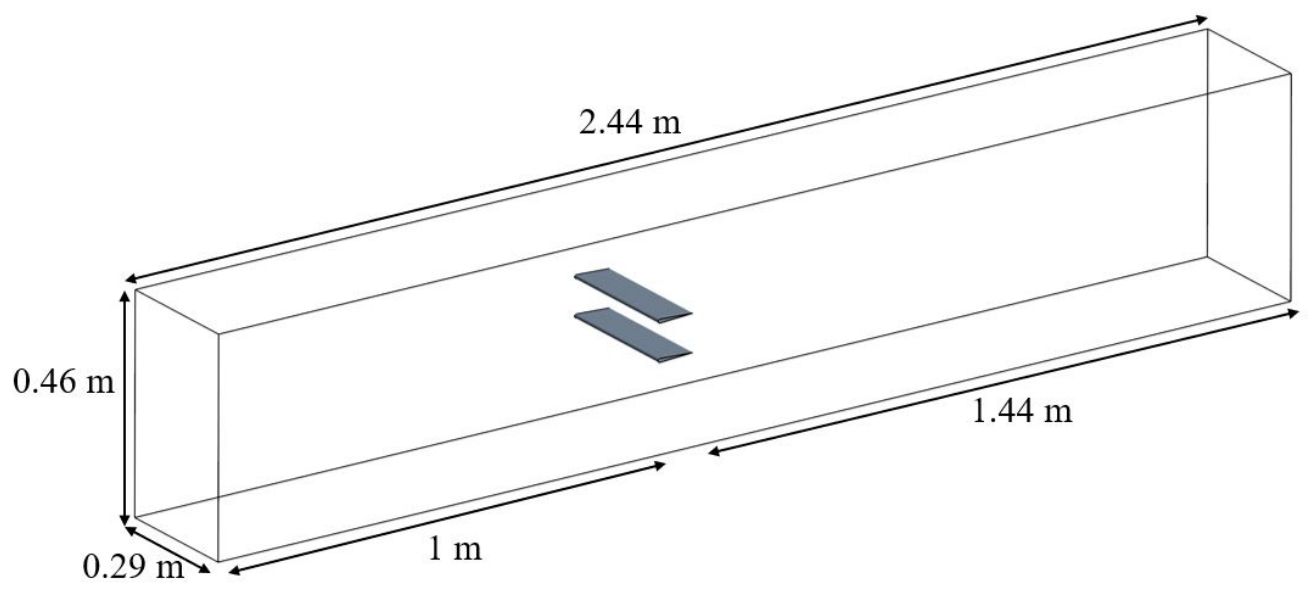

Figure 3.10: Pitching fin validation domain.

The inlet velocity was $0.06 \mathrm{~m} / \mathrm{s}$ with water as the working fluid. The Reynolds number was 4700, based off the swimming of a bluegill sunfish [39]. All simulations were run with a Strouhal number of 0.25 , where Strouhal number is found using Equation 3.5: 


$$
S t=\frac{f A}{U_{\infty}}
$$

$S t=0.25$ is a common Strouhal number seen in nature and corresponds to a reduced frequency of $k=2 \pi f C / U_{\infty}=2 \pi$ [17]. The angular displacement of the fins is given by Equation 3.6:

$$
\theta=\theta_{0} \sin \left(2 \pi t^{*}+\phi\right)
$$

where $\theta_{0}$ is the maximum pitching angle, $t^{*}=f t$ where $f$ is the flapping frequency and $t$ is time, and $\phi$ is the phase difference between foils. From this information, it was determined that $f=0.75 \mathrm{~Hz}$ and $\theta_{0}=7.2^{\circ}$. Each foil is pitched about its leading edge.

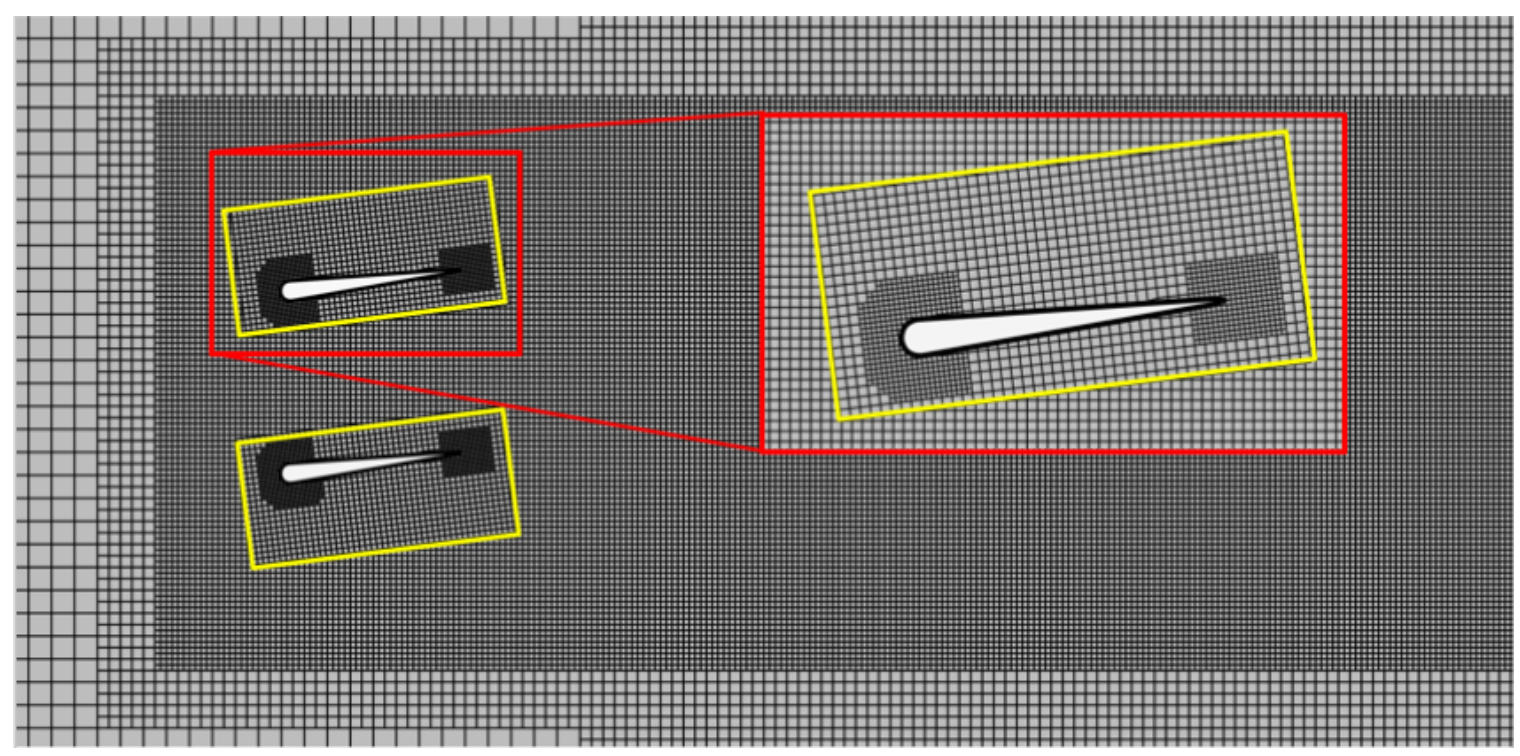

Figure 3.11: Hydrofoils mesh, with overset regions shown in yellow.

Due to the low Reynolds number, laminar flow was assumed. Meshing the fin motion was accomplished using two overset meshes, one for each fin. The mesh is shown in Figure 3.11. The total cell count was 5.56 million cells. The wake refinement 
area needed to be very large to capture the vortex streets.

\subsubsection{Hydrofoil Validation Results}

The results of interest were the coefficient of thrust and the vorticity scalar field for different phase differences. All scalar scenes were taken at mid-span of the foils. The coefficient of thrust is found using Equation 3.7. Figure 3.12 shows the comparison between the total $C_{T}$ results for increasing $\phi$, the oscillation phase differential between foils. $C_{T}$ is reported as $C_{T}^{*}=C_{T} / C_{T, s}$, where $C_{T, s}$ is the coefficient of thrust for a single pitching foil. $D^{*}=d / C$ is the spacing between foils, normalized with foil chord length. The general trend for both results is similar, however the CFD results start to overpredict the thrust as the phase differential is increased. The percent difference in results at $\phi=\pi$ is near $20 \%$.

$$
C_{T}=\frac{T}{\frac{1}{2} \rho U_{\infty}^{2} A}
$$

Figures 3.13 - 3.15 shows the comparison of vorticity fields in the near wake region. The experimental results were captured using particle image velocimetry (PIV). The vorticity is nondimensionalized such that $\omega^{*}=\omega C / U_{\infty}$. The shedding characteristics are qualitatively identical between the simulation and experimental results. One note is that the overset mesh is generating artificial vorticity, which is visible at the edges of the overset region. This seems to be a post-processing facet of STAR-CCM+ that incorrectly presents the vorticity field using the scalar blending function, since changing the contour style to 'automatic' removes this issue.

Because the coefficient of thrust trends are comparable, and the wake results are similar, the simulation is sufficiently validated. 


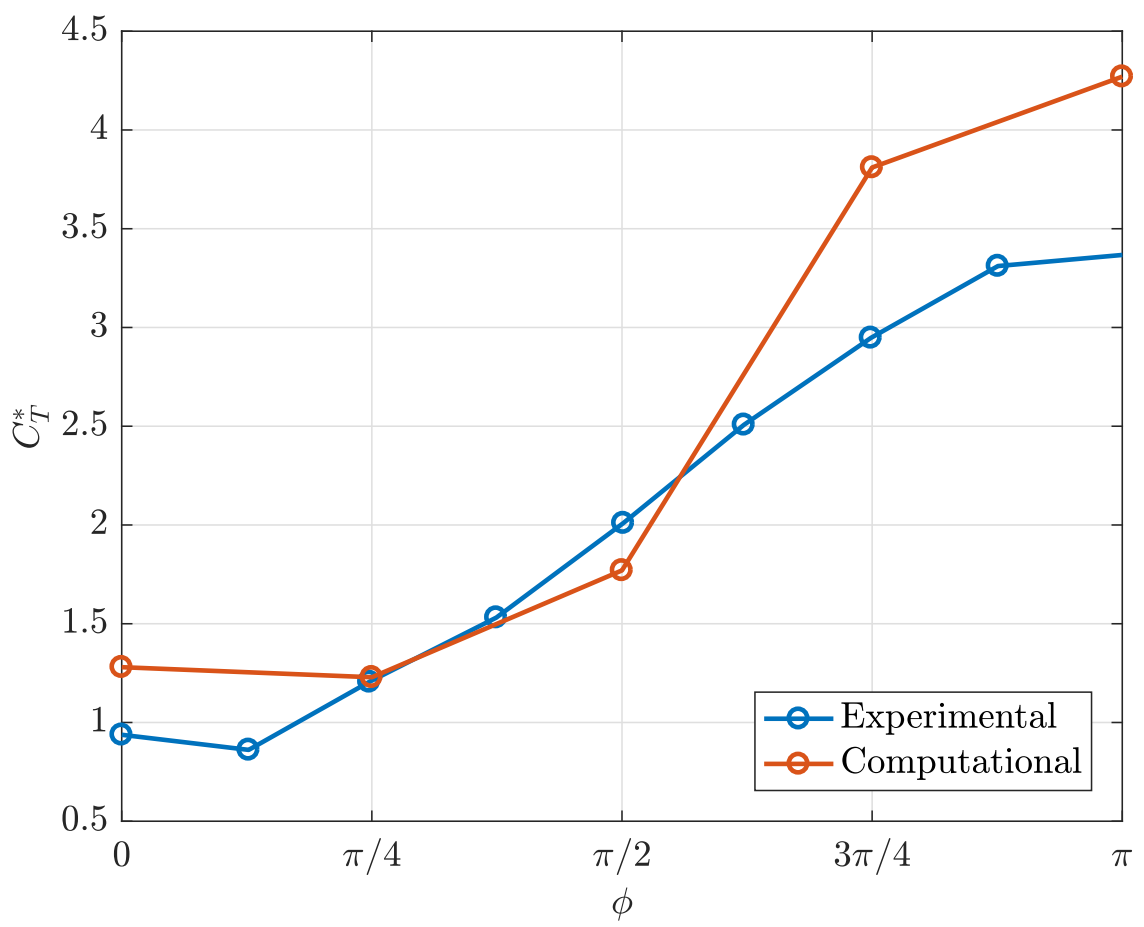

Figure 3.12: Coefficient of thrust versus phase differential $\phi$ for $D^{*}=0.5$.

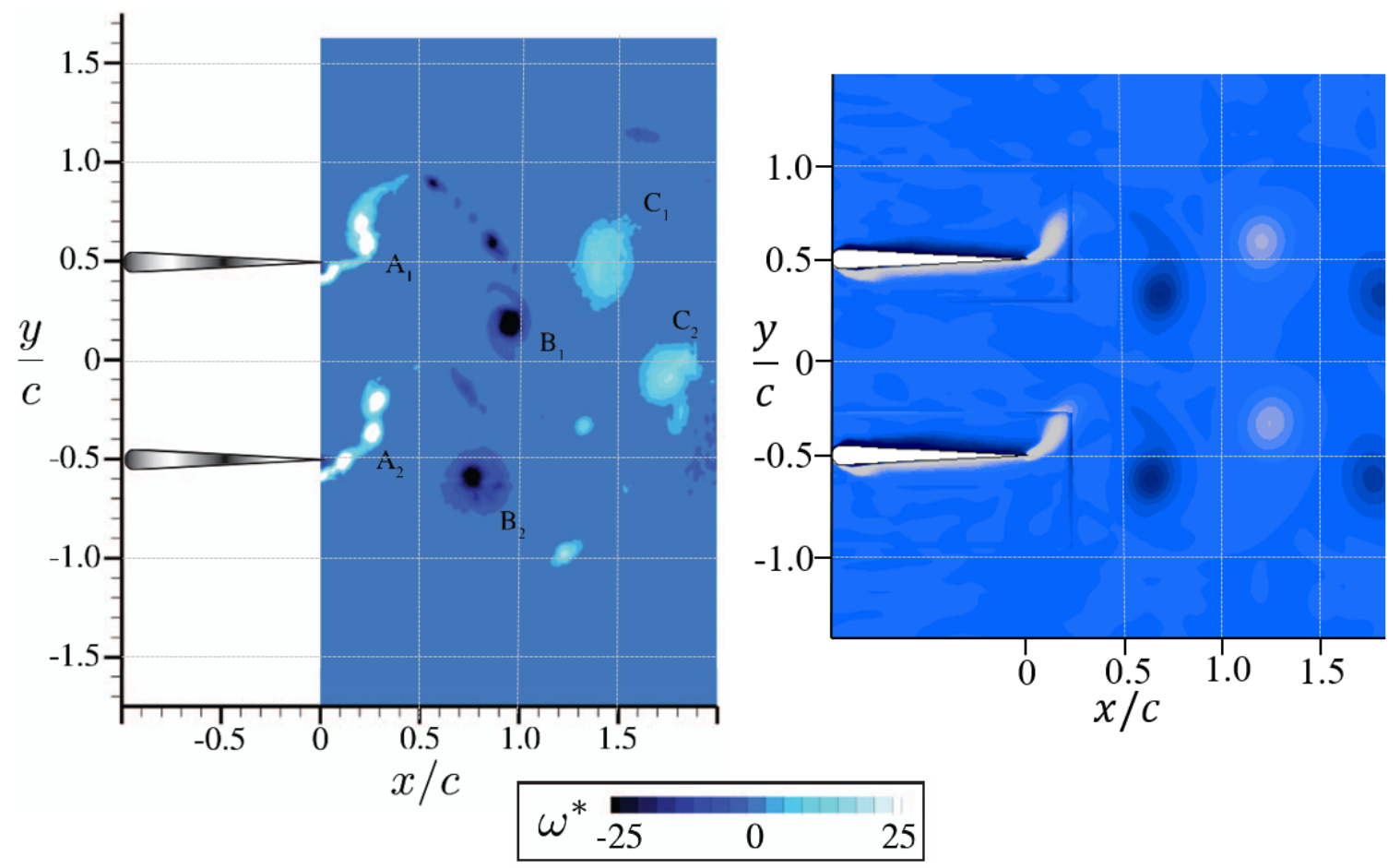

Figure 3.13: Comparison of experimental results to computational results for $\phi=0[17]$. 


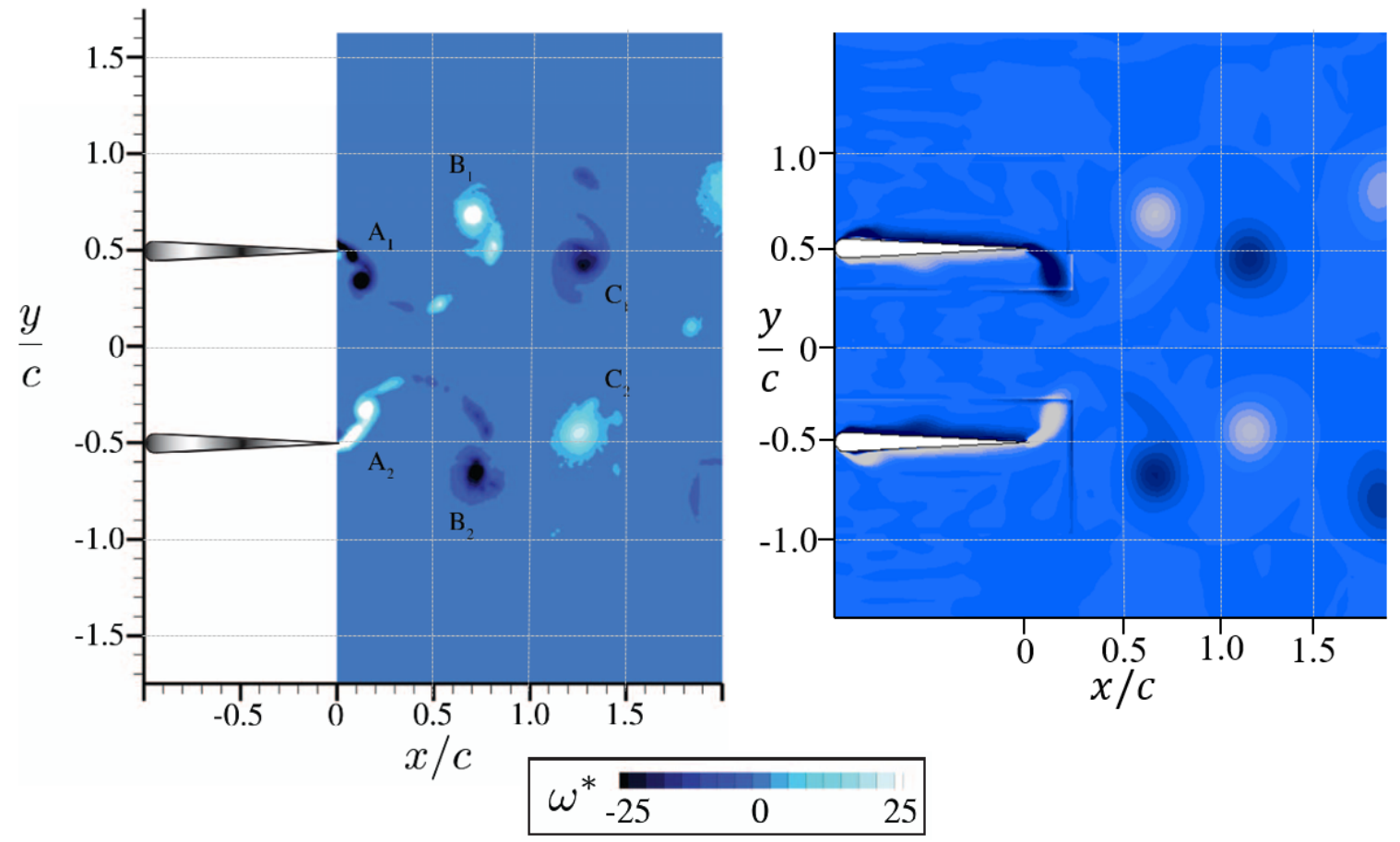

Figure 3.14: Comparison of experimental results to computational results for $\phi=\pi[17]$.

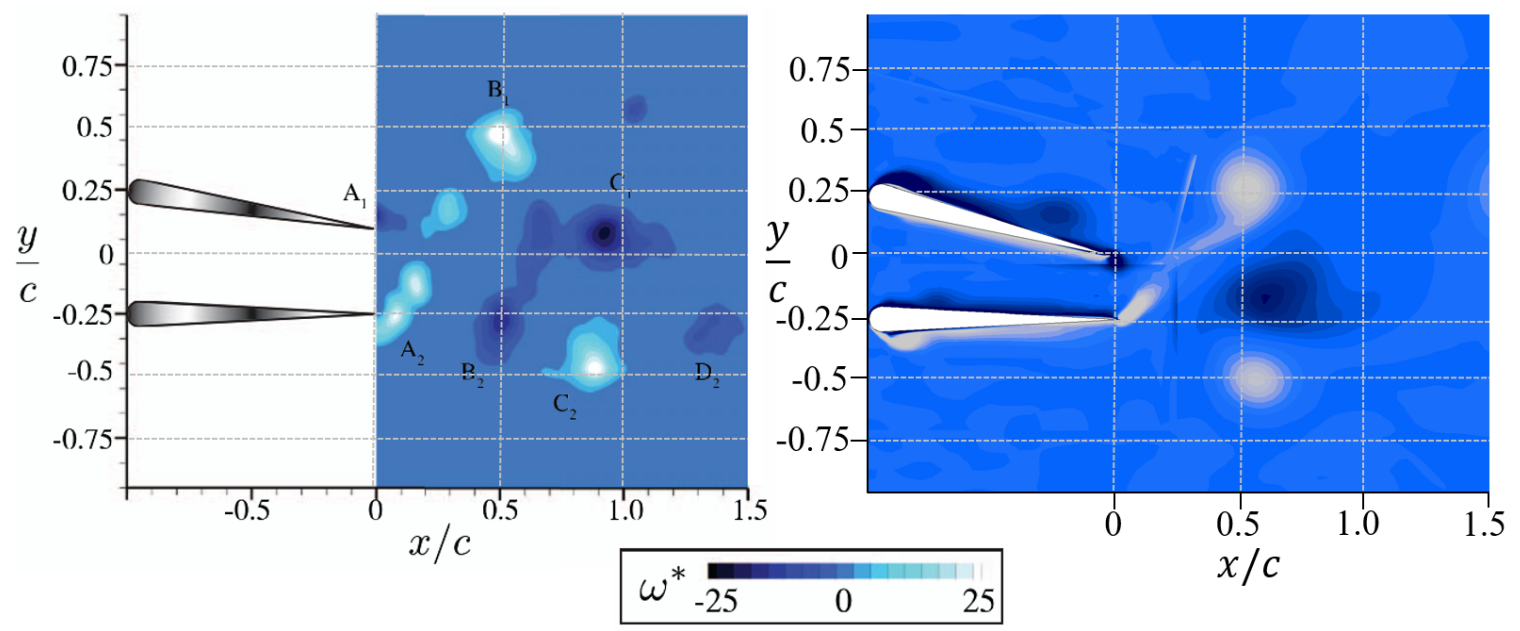

Figure 3.15: Comparison of experimental results to computational results for $\phi=\pi / 2[17]$. 
Chapter 4

FINAL SIMULATION SETUP AND RESULTS

\subsection{Setup}

\subsubsection{Model Geometry}

The final geometry is shown in Figures 4.1 and 4.2. The geometry is identical to the validated cases, however the dimensions are increased to better reflect the leopard seal proportions. The 6:1 prolate spheroid is no longer at an angle of attack and has a length of 2.98 meters, based off the leopard seal body length [12]. The foils are a chord length apart from each other. They are positioned directly downstream of the spheroid body, with the leading edges $0.1 C$ ahead of the spheroid trailing edge.

A grid convergence study was conducted on the spheroid model to determine the mesh quality needed to obtain accurate results without having unnecessarily long computation times. This study influenced the final mesh settings, which were pulled from the validation cases. A few modifications were made to better capture the boundary layer on the foils since the flow field is turbulent. The total cell count is 5.8 million. Mesh detail is shown in Figure 4.3.

\subsubsection{Physics Settings}

The time step was lowered from the validation case value of 0.005 to $0.0025 \mathrm{~s}$. This was done because the frequency corresponding to $S t=0.5$ is significantly faster than the frequency for the validation case, which was run at $S t=0.25$. The STAR$\mathrm{CCM}+$ manual recommends having between 50 - 100 time steps per cycle for periodic

flows [40]. A timestep of $0.0025 s$ results in 72 time steps for the $S t=0.5$ case, and 


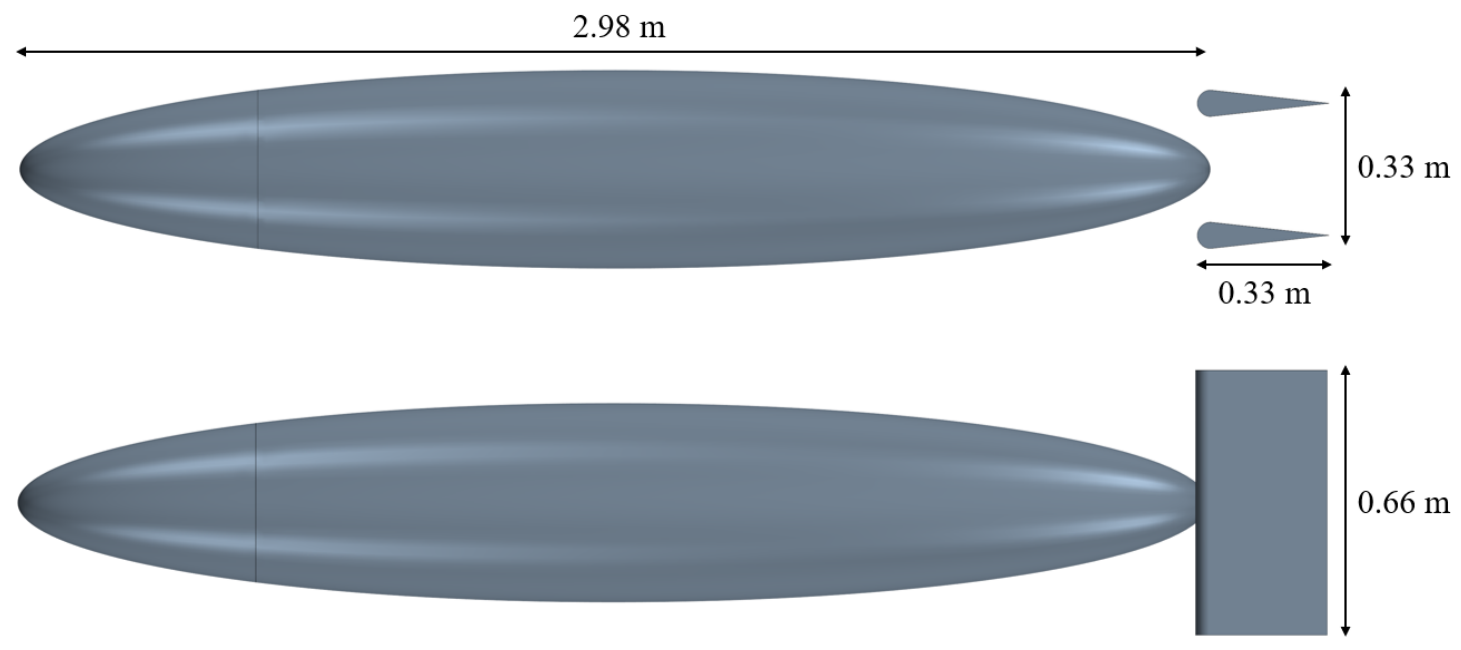

Figure 4.1: Final model geometry.

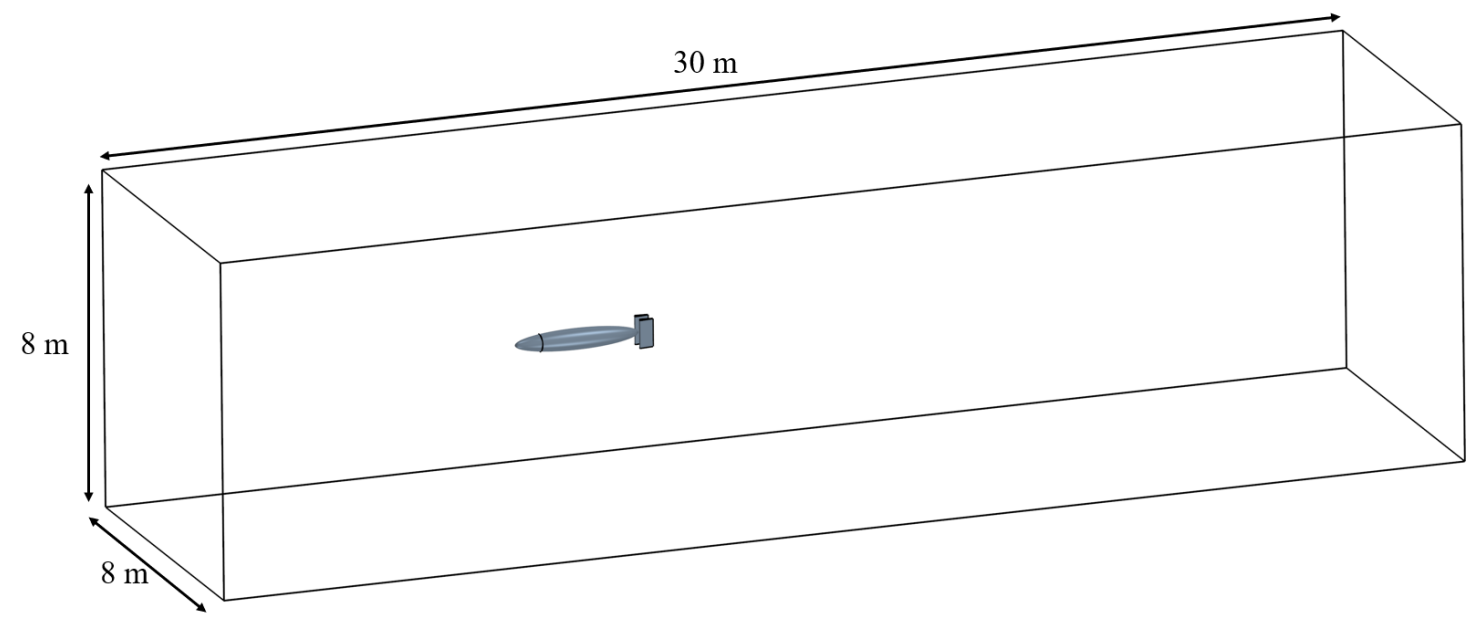

Figure 4.2: Final domain dimensions. 


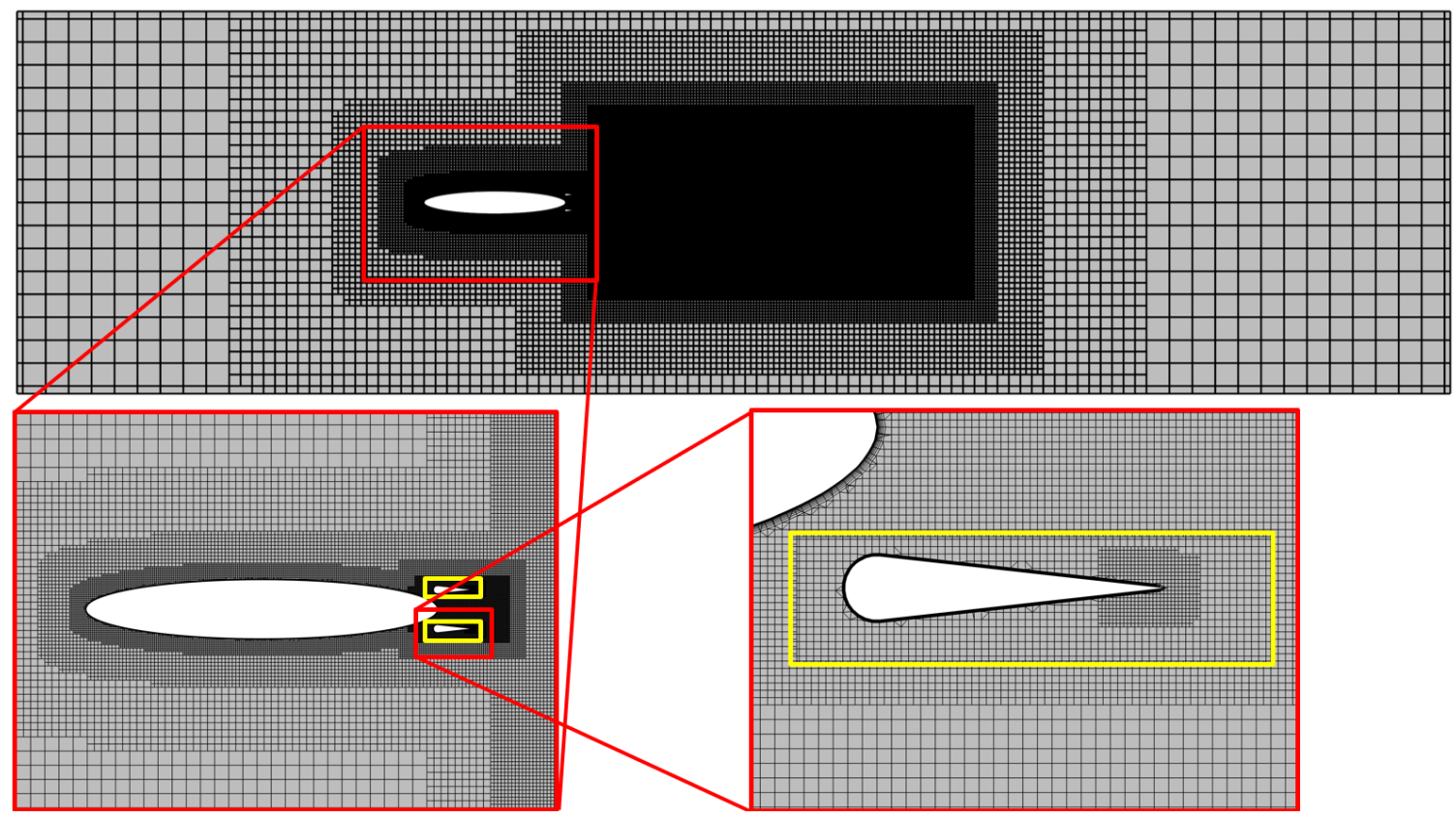

Figure 4.3: Final mesh, with overset meshes shown in yellow.

more for the lower frequency cases. Modeling motion with an overset mesh and a first order temporal discretization scheme requires the timestep be small enough such that the maximum movement is the smallest cell in the overlapping zone. If a second order discretization scheme is used, the maximum movement per timestep decreases to half the smallest cell size. It was unrealistic to use such a small timestep (or, equivalently, to sufficiently increase the mesh size) so the first order temporal discretization scheme was chosen.

The maximum pitching angle was selected to be 10 degrees, slightly higher than that of [17]. The maximum angle must be relatively small (ie, below the stall angle) to generate thrust, otherwise the wakes of both foils will destructively interact [9] and produce negative thrust. The prescribed motion is described by Equation 3.6. The inlet velocity is $1.25 \mathrm{~m} / \mathrm{s}$ and was chosen to yield a Reynolds number of $4.2 \times 10^{6}$ based on the spheroid length, matching the spheroid validation case. The chosen velocity is a realistic leopard seal speed, within the range of speed observed in [41]. 
Like the prolate spheroid validation case, the boundary layer was artificially tripped at $x / L=0.2$.

\section{$4.2 \quad$ Results}

Two series of simulations were conducted to investigate how an upstream body impacts the thrust generation and flow field of tandem hydrofoils. One set was run with the body, and one was run without. The simulations were run for a range of $S t$ values $(0.2-0.5)$ and phase differences $(0-\pi)$ in increments of 0.1 and $\pi / 4$, respectively. Phase differences greater than $\pi$ were not run because the results are symmetrical.

The body simulations were run for ten cycles: nine cycles to reach a pseudo-steady state, and one cycle to obtain data. Vorticity is nondimensionalized as $\omega^{*}=\omega C / U_{\infty}$ where $\mathrm{C}$ is the foil chord length. Thrust is nondimensionalized as $C_{T}$, using Equation 3.7, where $A$ is the foil area. All scalar scenes show a top-down view of the model. The scalar scenes are on a cut plane at mid-span of the body and foils.

Figures 4.4 shows the pitching sequences and accompanying thrust trend for $\phi=0$. For the $\phi=0$ case the thrust generation is nearly identical since the foils are pitching together, however the inside foil (the top foil before $f t=1 / 2$ and the bottom foil after) produces a slightly lower amount of thrust due to the outside foil inducing negative velocity on it.

Each foil sheds two vortices per cycle. When $\phi=0$, the vortices are shed in pairs. The first pair of like-signed vortices shed as the foils approach $\theta=\theta_{\max }$, at $f t=1 / 3$. The following vortices form immediately after the first pair sheds, and have the opposite sign as the previous pair. The second pair sheds half a cycle later as the foils approach $\theta=-\theta_{\max }$. The vortices dissipate quickly and propagate outwards and downstream through the wake. Figure 4.5 shows both vortex pairs shedding. The shedding event corresponds to the point when the inside and outside foils switch, or 


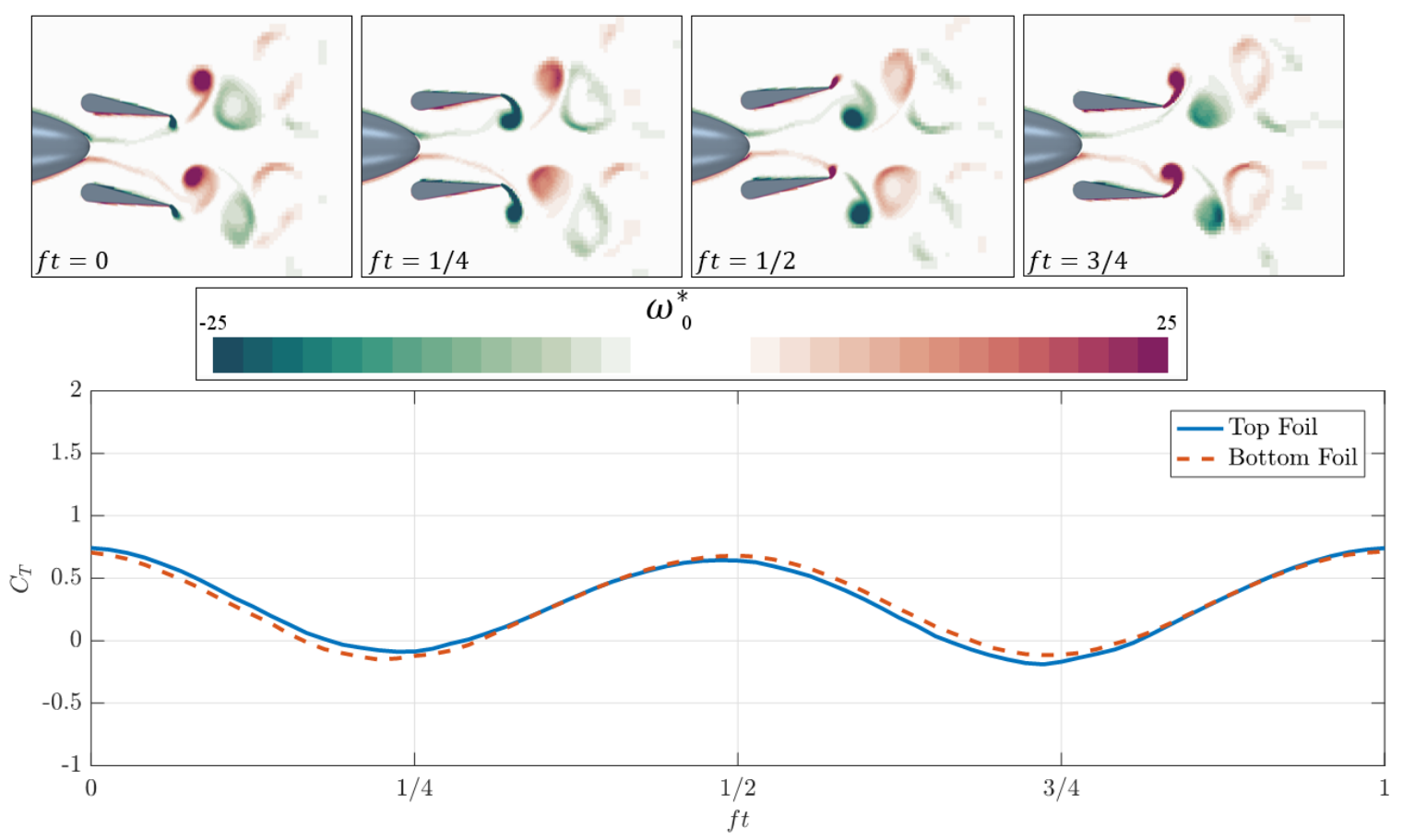

Figure 4.4: Pitching sequence and thrust trend for $\phi=0$ and $S t=0.5$.

when the instantaneous thrust is equal for both foils.

Figure 4.6 shows the pitching sequence for $\phi=\pi$, when the foils are pitching completely out of phase. but since the shedding events are timed identically (and are just of the opposite magnitude) the thrust generation is equal.

When the foils are pitching out of phase $(\phi=\pi)$ the timing is identical to $\phi=0$ and the vortices are still shed in pairs, but they are now counter-rotating as a result of the foils pitching in different directions. When the foils pitch away from each other, the vortices are rotating inwards. As the foils pitch towards each other the vortices form on the outside of the foil, rotating away from the foil, stretching themselves. Both shedding occurrences are shown in Figure 4.7.

For the intermediate case of $\phi=\pi / 2$, detailed in Figure 4.8, the phase difference causes the thrust trend to shift forward by a quarter of a cycle such that the peak of the leading foil aligns with the trough of the trailing foil, and vice versa. 

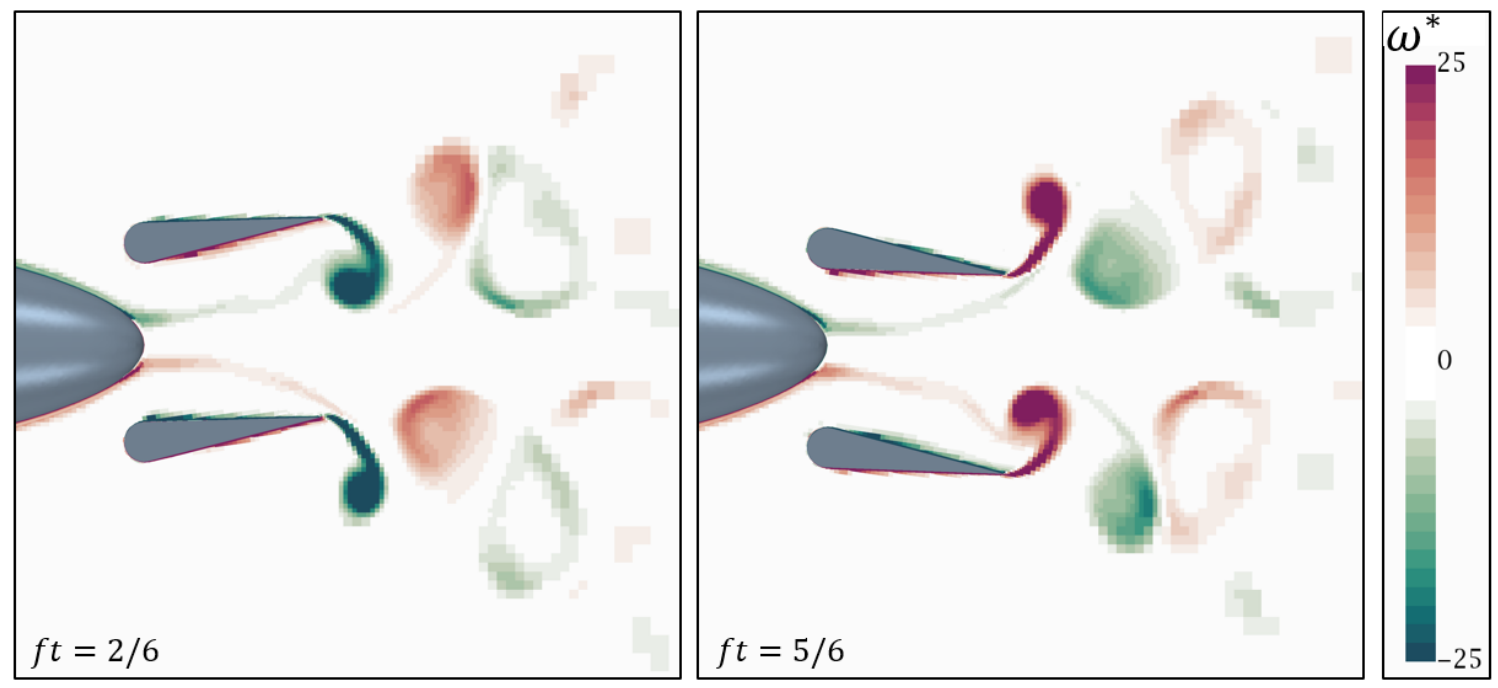

Figure 4.5: Shedding events for $\phi=0$ at $S t=0.5$.
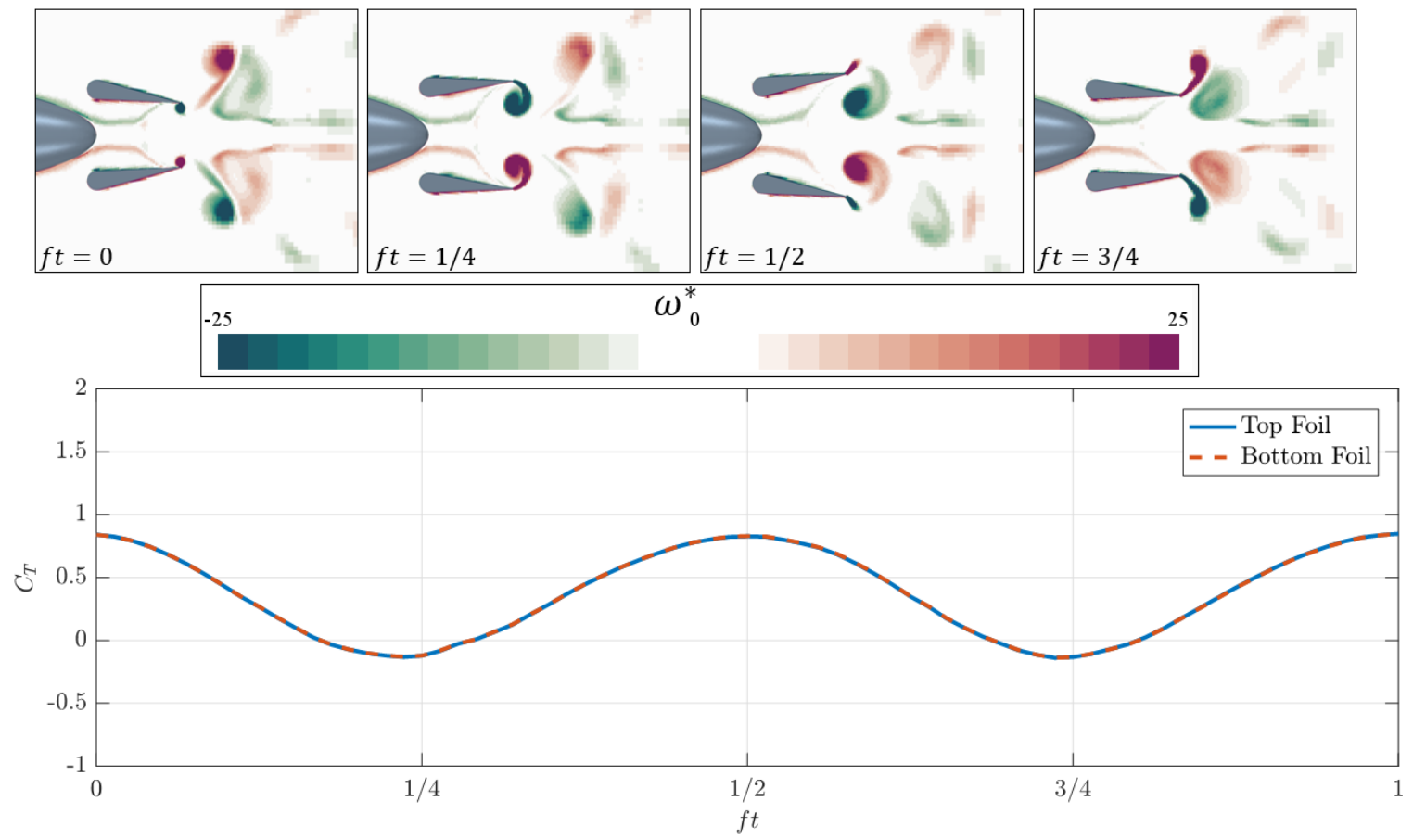

Figure 4.6: Pitching sequence and thrust trend for $\phi=\pi$ and $S t=0.5$. 

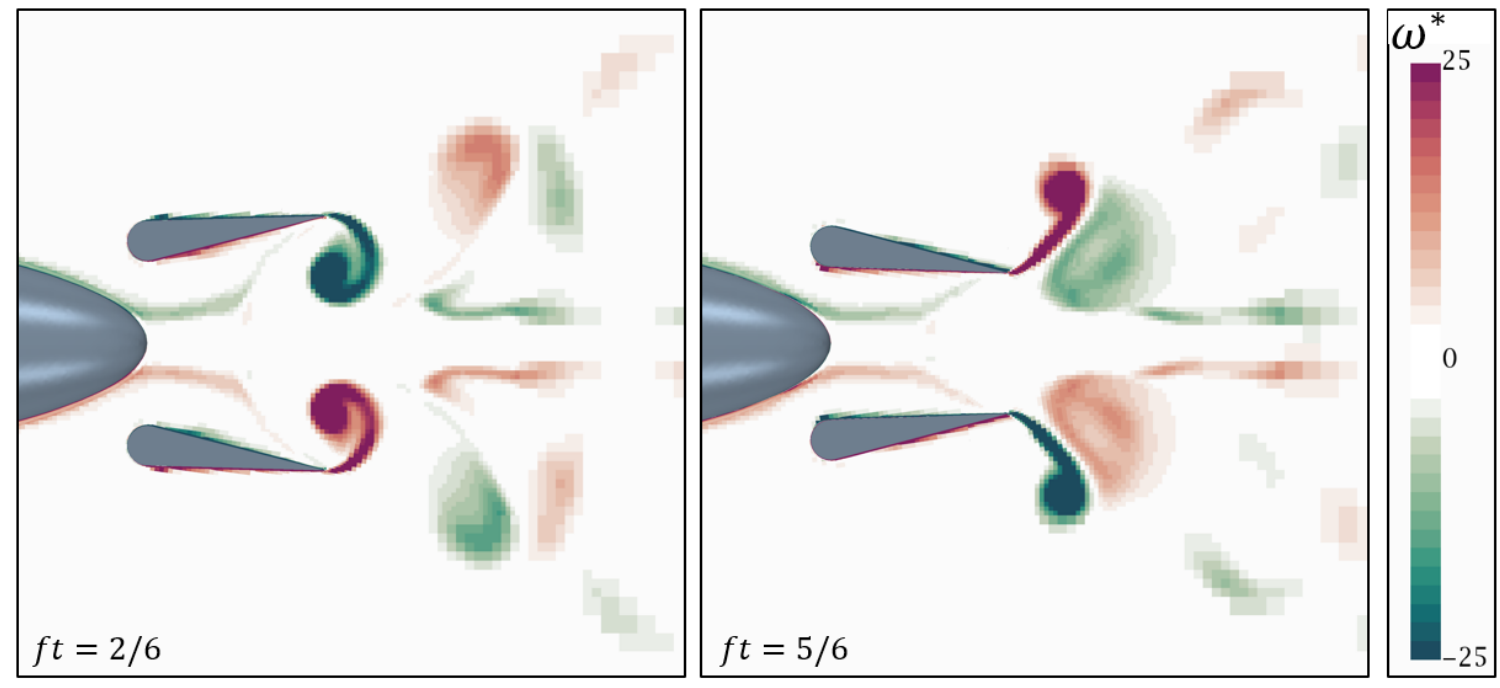

Figure 4.7: Shedding events for $\phi=\pi$ at $S t=0.5$.
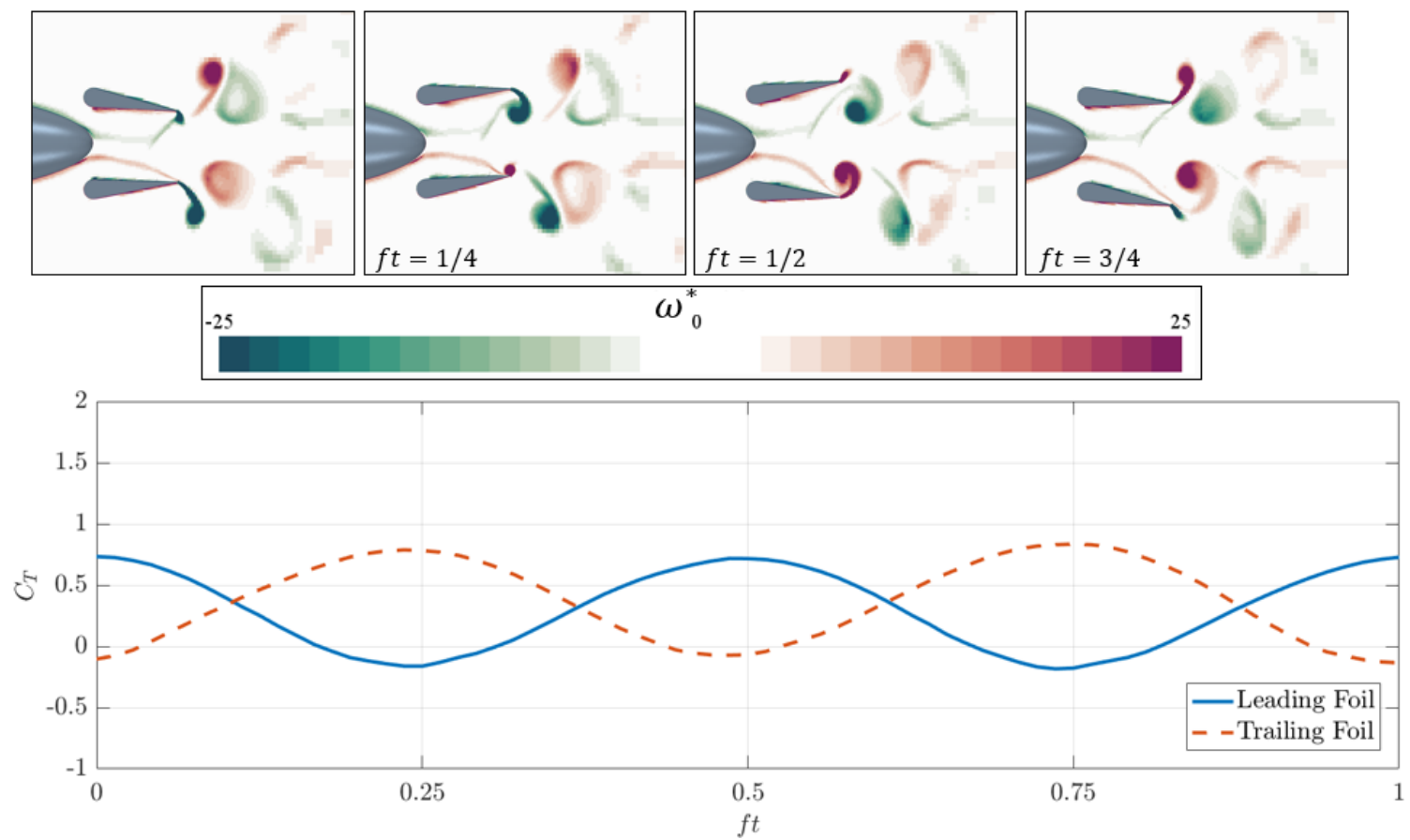

Figure 4.8: Pitching sequence and thrust trend for $\phi=\pi / 2$ and $S t=0.5$. 

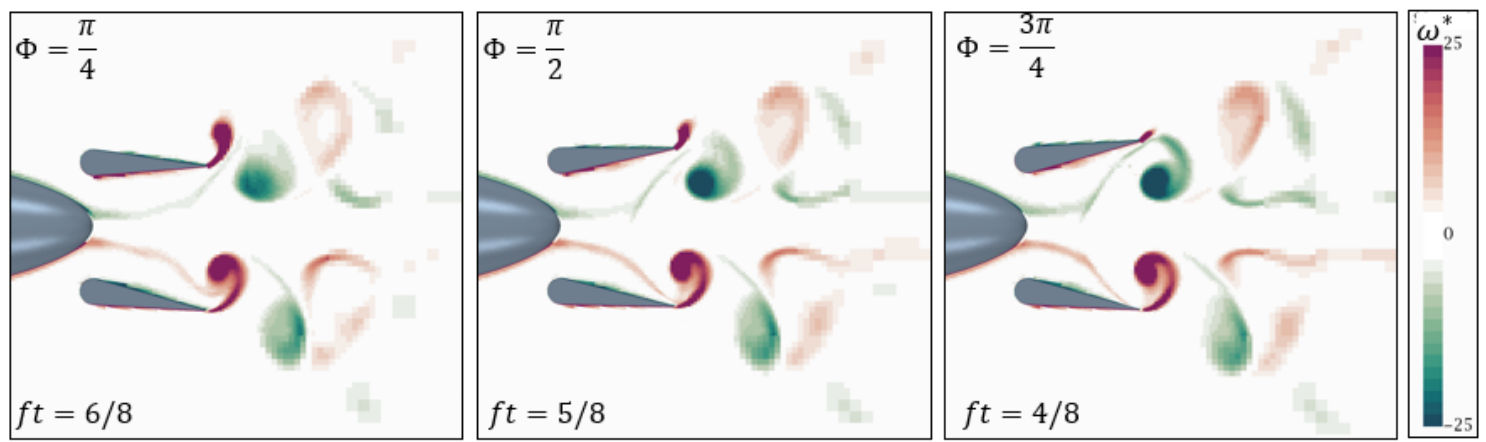

Figure 4.9: Comparison of shedding event for intermediate phase differences. The leading foil is the bottom foil.

The shedding behavior changes more at intermediate phase differences than at $\phi=0$ or $\phi=\pi$. The trailing foil thrust trend (the foil with no $\phi$ term in the motion equation, and is the top foil in all presented images) is identical in shape for all phase differences, indicating that at the presented foil spacing of one chord length there is minimal interference between the two wakes. The leading foil thrust trend is shifted forward in time as a result of adding the phase difference.

An increase of $\pi / 4$ in the phase difference advances the shedding event by $f t=$ 1/8, shown in Figure 4.9. At $\phi=\pi / 4$ the leading foil sheds a like-signed vortex preceding the trailing foil. At $\phi=\pi / 2$ the leading foil shedding occurs when the trailing foil is at its farthest away from a shedding event, and at $\phi=3 \pi / 4$ the leading foil sheds a counter-rotating vortex after the trailing foil. This has implications for the thrust generation. However, the wake structure is similar for each case, only varying in the magnitude of the downstream vortices.

The thrust trend for the trailing foil is shown in Figure 4.10. While the amplitude varies with phase difference, the shape remains unchanged and is always sinusoidal. The leading foil thrust generation sees a shift in the thrust trend, as illustrated in Figure 4.11, which causes the leading foil to generate a greater amount of thrust at intermediate phase differences. Increasing the phase difference by $\pi / 4$ advances the peak in thrust generation by roughly $f t=0.125$ which agrees with the behavior 


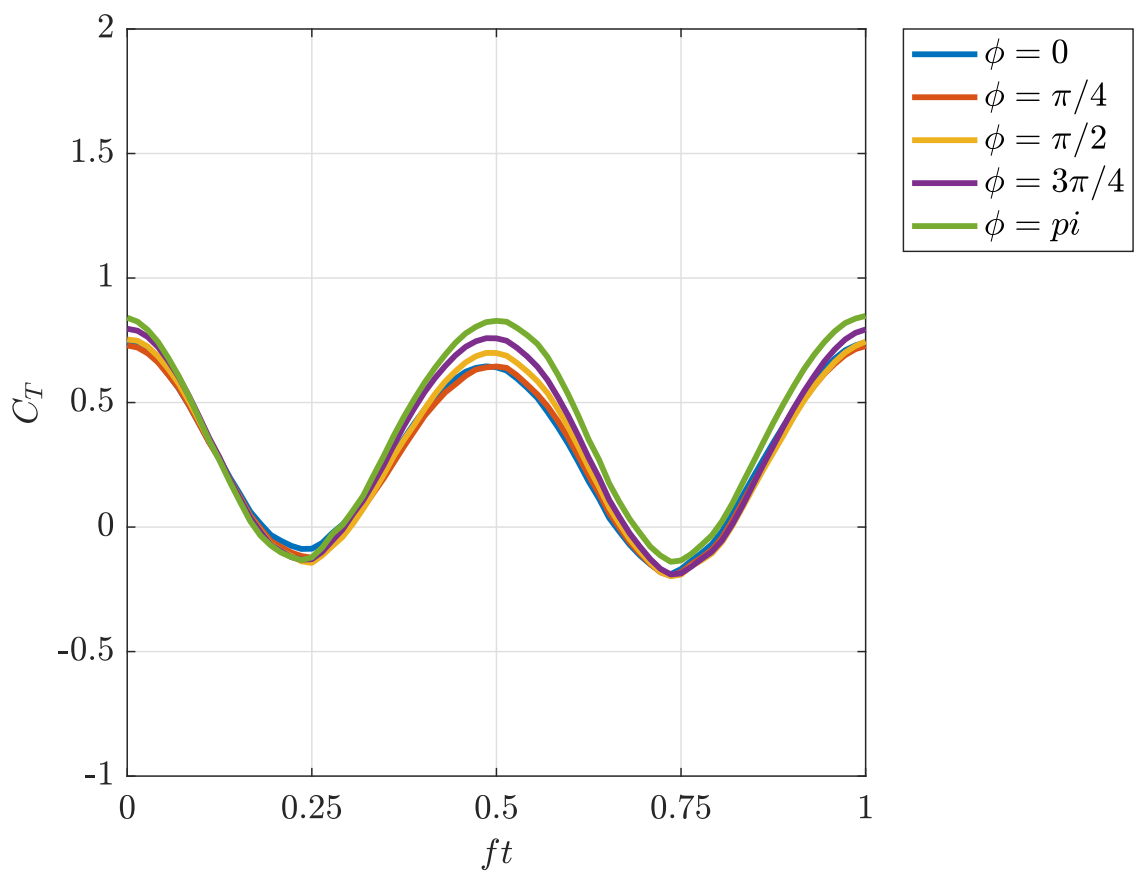

Figure 4.10: Trailing foil thrust trend for increasing $\phi, S t=0.5$

exhibited in Figure 4.9. When the foils are pitching out of phase this results in an offset of $f t=0.5$, which causes the second peak to line up with the first thrust peak in the cycle, producing an identical pattern as the $\phi=0$ case.

At intermediate phase differences the leading foil produces large troughs in thrust that occur $f t=1 / 12$ before the second vortex is shed. However, the local minimum trough (that in Figure 4.11 occurs prior to the deeper trough for $\phi=\pi / 2$ and $\pi 4$ and after for $3 \pi / 4$ ) coincides with a shedding event. The large uptick and peak in thrust occurs when the leading foil is close to $\theta_{\max }$, which could suggest that higher pitching angles lead to an increased thrust.

The combined and separated time averaged thrust results are shown in Figure 4.12 and Figure 4.13, respectively. The thrust is seen to increase monotonically with Strouhal number, albeit with diminishing returns. Raising the Strouhal number from 0.2 to 0.3 triples the thrust, and from 0.4 to 0.5 there is only a $67 \%$ increase in thrust across all phase differences, still a significant amount. When the phase difference 


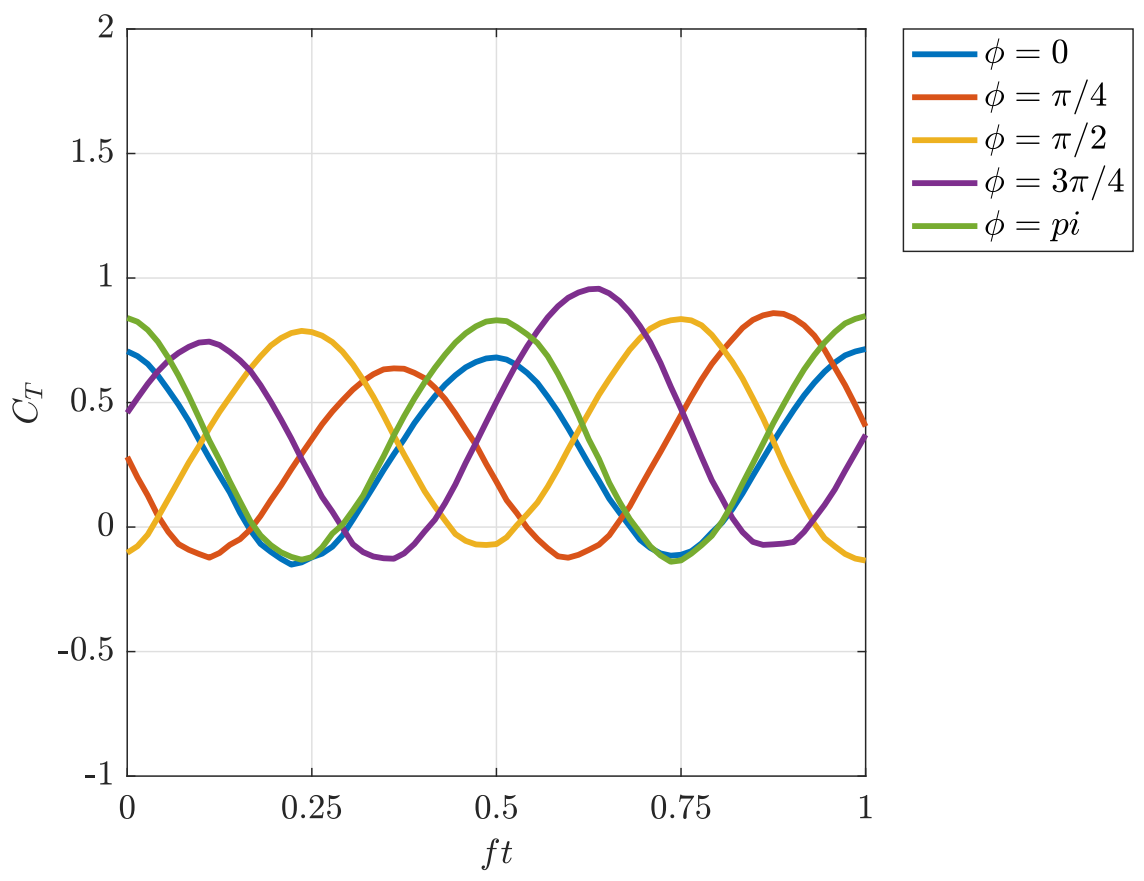

Figure 4.11: Leading foil thrust trend for increasing $\phi, S t=0.5$

is 0 or $\pi$ the average thrust generated by both foils is identical. At $\phi=0$ this is because the foils have the same motion and the wakes do not interact with each other, causing destructive interference. At $\phi=\pi$ the foils are mirrored across the centerline, producing the highest amount of average thrust. To determine the error in the time averaged thrust, a mesh study was conducted using Equation 3.2 for the $S t=0.5$ and $\phi=\pi$ case. The error in one direction was found to be 0.016 .

At intermediate phase differences there is an imbalance in the thrust produced by the separate foils, which increases with Strouhal number. The leading foil always produces the majority of the total thrust, and peaks at $\phi=3 \pi / 4$. This contrasts with the trailing foil behavior, which peaks at $\phi=\pi$ and has its lowest value at $\phi=\pi / 4$, when the leading foil is an eighth of a cycle ahead of it.

The largest disparity in thrust generation occurs at $\phi=\pi / 2$. At $S t=0.5$ the leading foil produces $21.7 \%$ more thrust than the trailing foil. This could result in an angled thrust vector which could be useful for maneuvering purposes. 


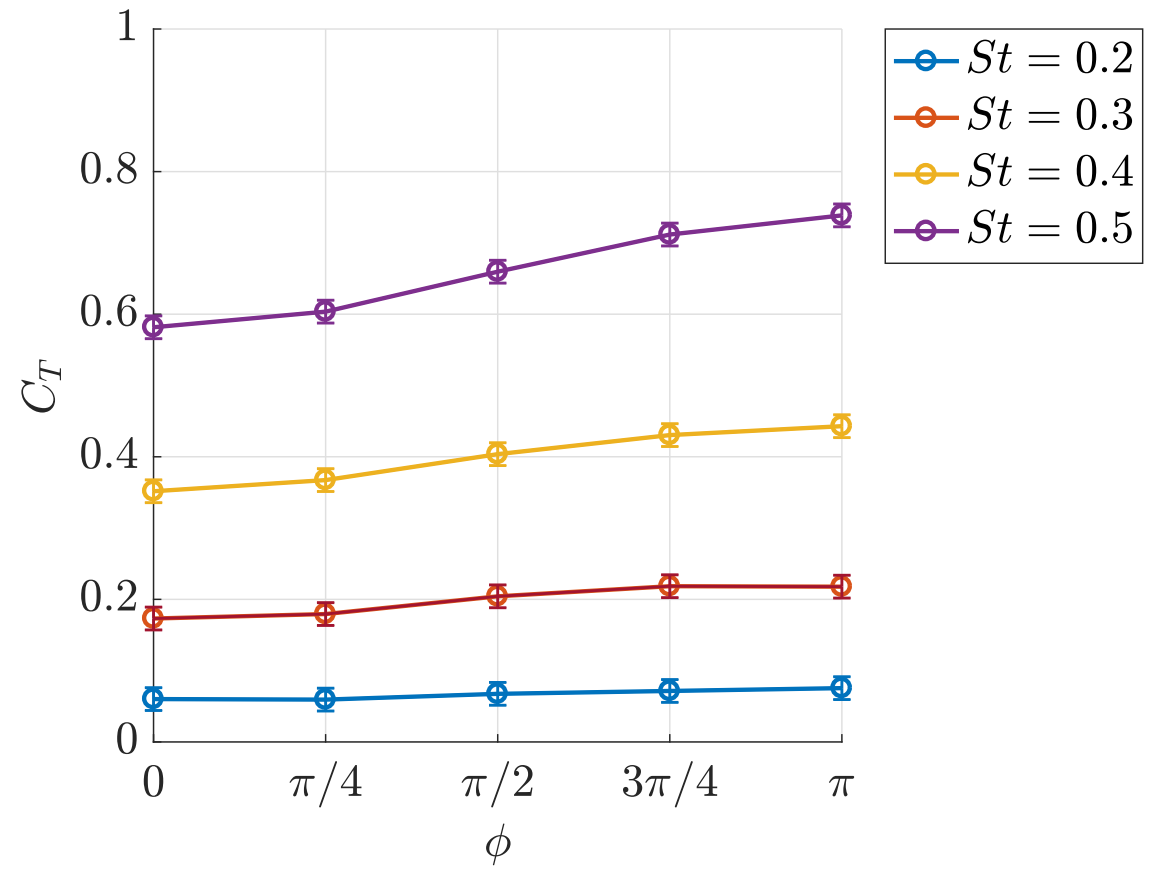

Figure 4.12: Combined time averaged thrust results as a function of phase difference $\phi$.

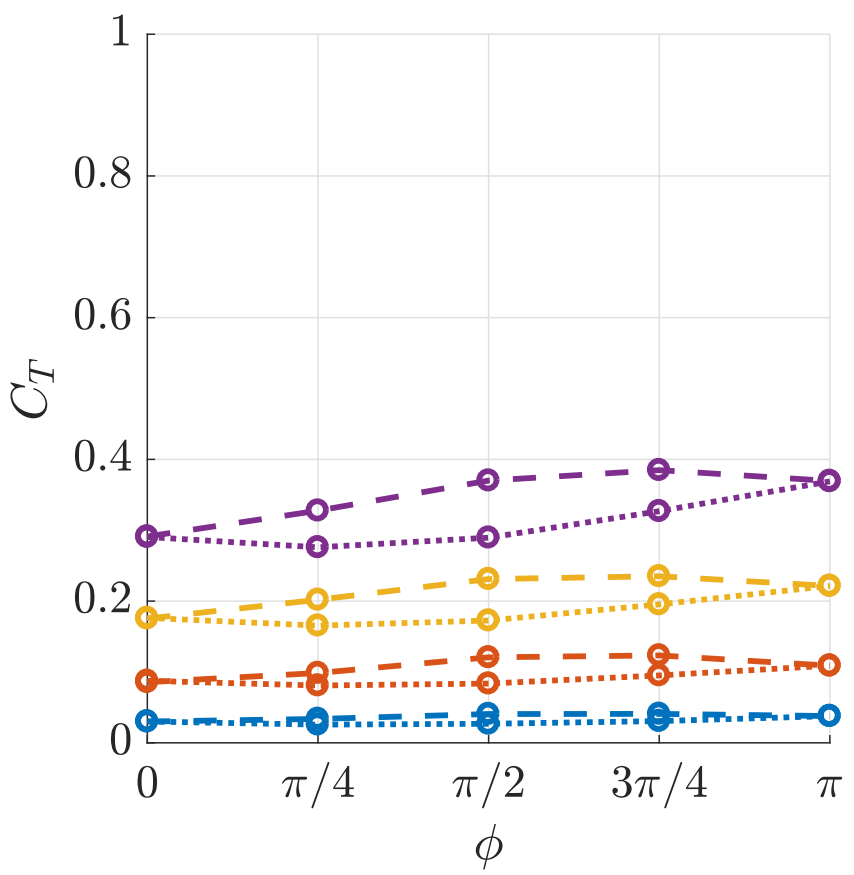

$$
\begin{aligned}
& \text {.०. } S t=0.2 \\
& \text { - ० - } S t=0.3 \\
& \because \mathrm{O}: S t=0.4 \\
& \text {. - } \cdot S t=0.5
\end{aligned}
$$

Figure 4.13: Leading and trailing foil time averaged thrust results as a function of phase difference $\phi$. The dotted line is the trailing foil and the dashed line is the leading foil. 

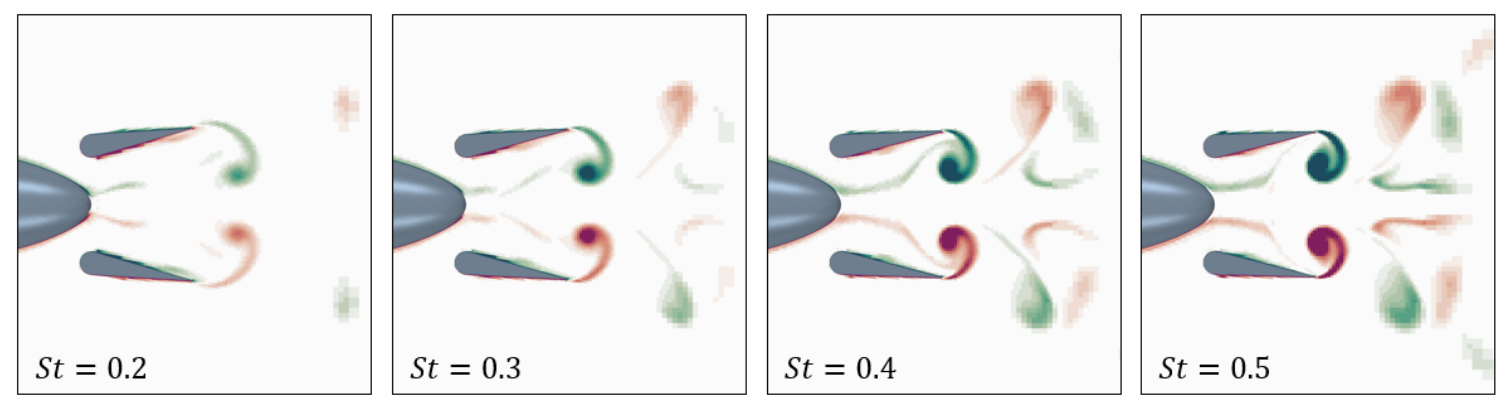

Figure 4.14: Differences in vortices and wake for different Strouhal numbers at $\phi=\pi$ and $f t=0.25$.

At $S t=0.2$ the lowest value is from the $\phi=\pi / 2$ case. For the medium Strouhal numbers the lowest value is $\phi=\pi / 4$, and at $S t=0.5$ it is at $\phi=0$. Breaking these results up into the separate foils, the trailing time averaged results do follow a trend of reaching a minimum at $\phi=\pi / 4$ before increasing until $\phi=\pi$. Interestingly, the thrust generation is identical for $\phi=0$ and $\phi=\pi / 2$.

The leading foil behavior is more complicated. For $S t=0.5$ the leading foil thrust increases with $\phi$. At $S t=0.4$ the thrust does not increase between $\phi=\pi / 4$ and $\pi / 2$, and for $S t=0.3$ and $S t=0.2$ the thrust generation decreases from $\phi=0$ to $\phi=\pi / 2$, where there is a small amount of negative thrust being produced. From $\phi=\pi / 2$ the thrust increases significantly to its maximum value at $\phi=\pi$.

Figure 4.14 shows a shedding event for each tested Strouhal number with $\phi=\pi$. As expected, the vorticity magnitude is higher for the upper Strouhal number cases. Due to the slow pitching motion the lower frequency vortices do not coil up upon formation and are instead shed as strands of lower magnitude vorticity, which dissipate significantly faster. This results in a significantly reduced thrust for the low Strouhal number cases.

The differences in thrust production of the foils are shown qualitatively in the pressure scalar scenes shown in Figure 4.15. As expected, the $\phi=\pi$ foils see identical pressure distributions as the pitching motion is identical across the centerline. For 


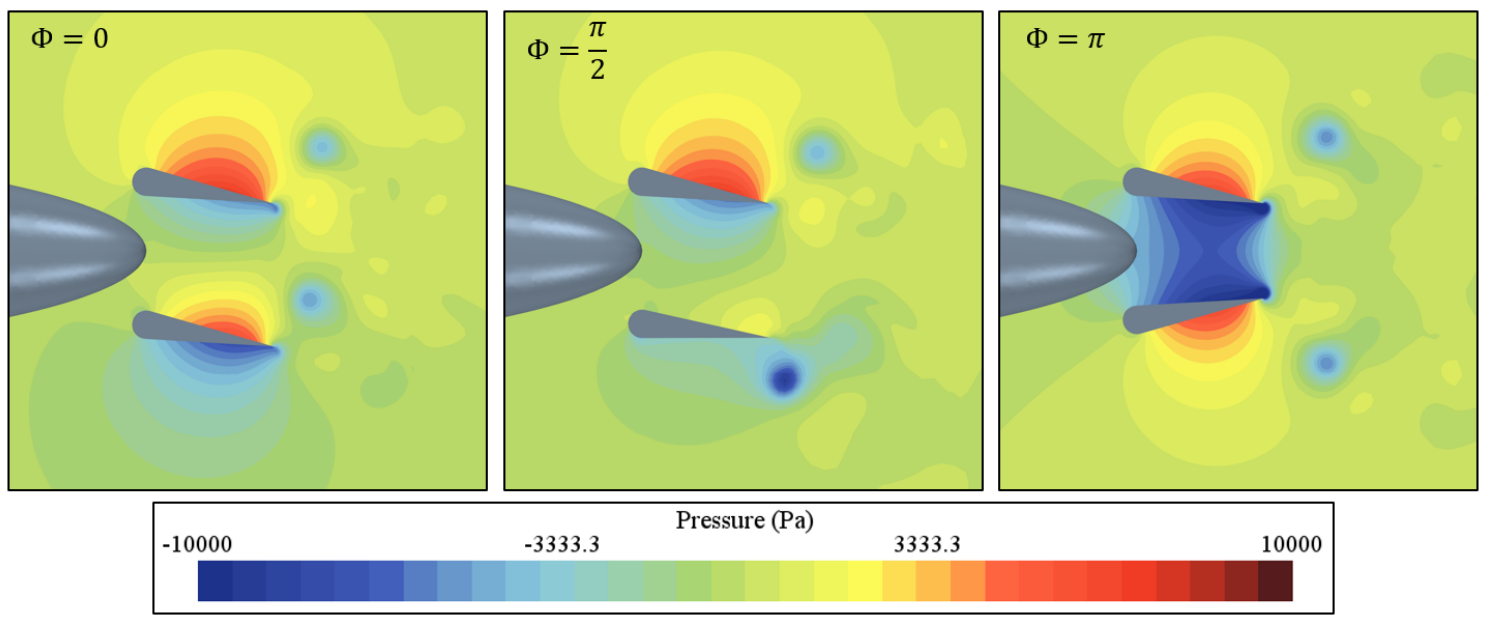

Figure 4.15: Pressure scalar scenes for $S t=0.5$ at $f t=1$.

$\phi=0$ the bottom fin sees lower pressures than the top, however when the foils pitch in the opposite direction, the reverse will be true, resulting in nearly-equal time averaged thrust values. For $\phi=\pi / 2$ the top foil is producing the same amount of thrust as it did when the foils were in-phase, but the bottom foil pressure distribution is significantly smaller than the in- and out of- phase cases.

\subsection{Comparison to no body results}

With regards to thrust data, the pitching foils with no upstream body closely resemble the values with the body, though the addition of the prolate spheroid gives a nearnegligible (but universal) increase in thrust. All simulation comparisons are shown in Figure 4.18.

Qualitatively the wake is similar, but the area between the foils is different. Low magnitude vorticity from the body is seen interacting with the vortex generation process. The body vorticity is pulled downstream by the pitching motion. When the vortex sign and body vorticity sign align, the body vorticity attaches to the growing vortex. This results in a vortex that is larger in size than the ones created 

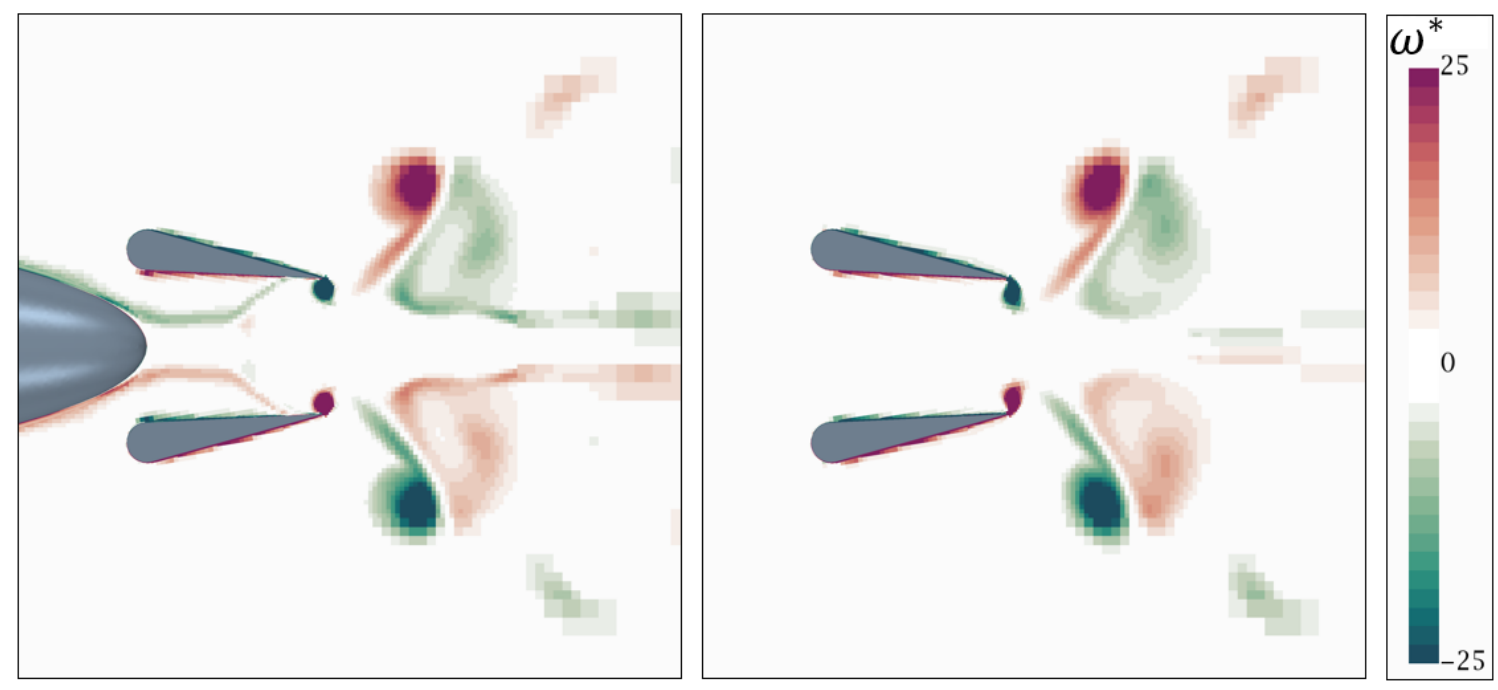

Figure 4.16: Comparison of vorticity scenes for $S t=0.5$ and $\phi=\pi$ when the vortex magnitude aligns with the body magnitude.

by the pitching foils without the body. Figure 4.16 shows the comparison when the vortex and body vorticity signs align, and Figure 4.17 shows when they are opposite. The body vorticity does not appear to destructively interact when the vortices being generated do not align.

The low vorticity magnitude shedding off the spheroid does not cause a significant change in the vortex shedding magnitude. For the $S t=0.5$ case there was a less than $3 \%$ difference in the maximum vorticity magnitude of the last two shed vortex pairs for any phase difference. Neither geometry had consistently higher vorticity than the other. 

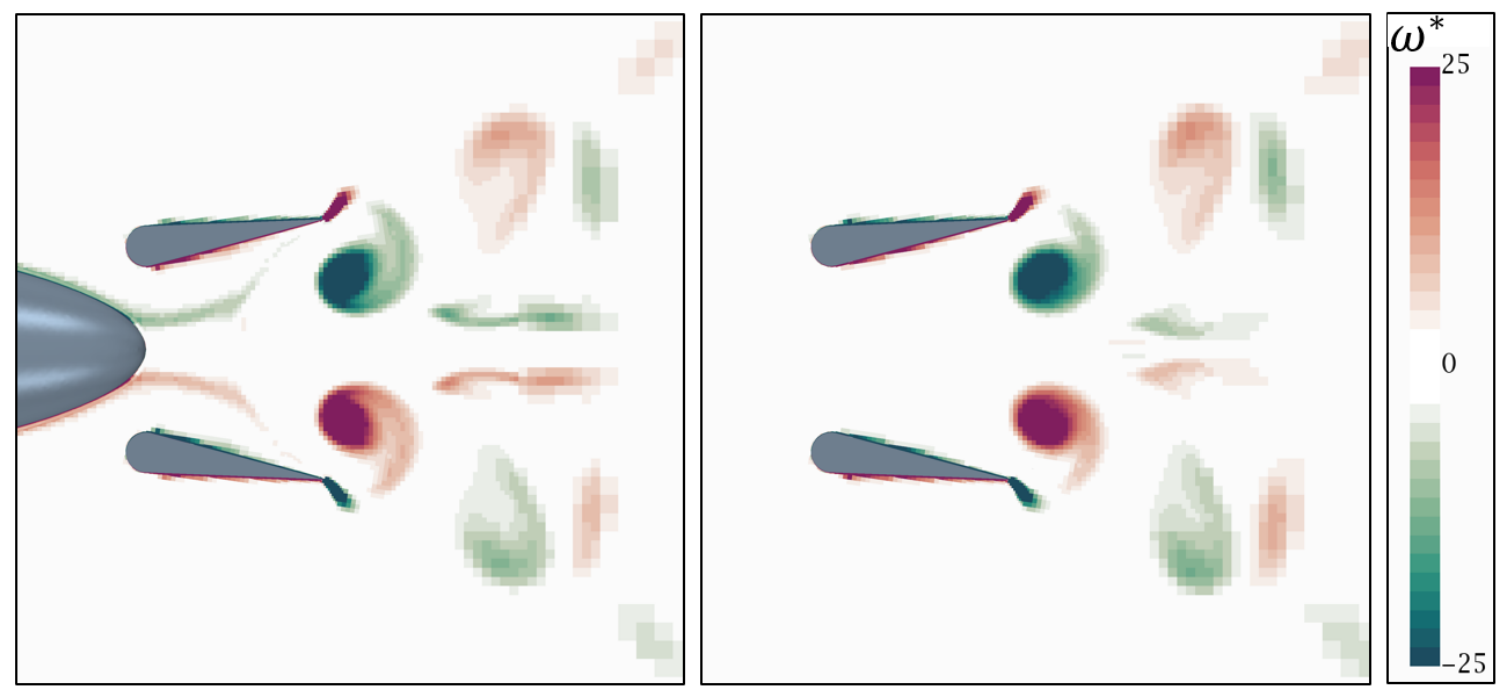

Figure 4.17: Comparison of vorticity scenes for $S t=0.5$ and $\phi=\pi$ when the vortex magnitude does not align with the body magnitude.

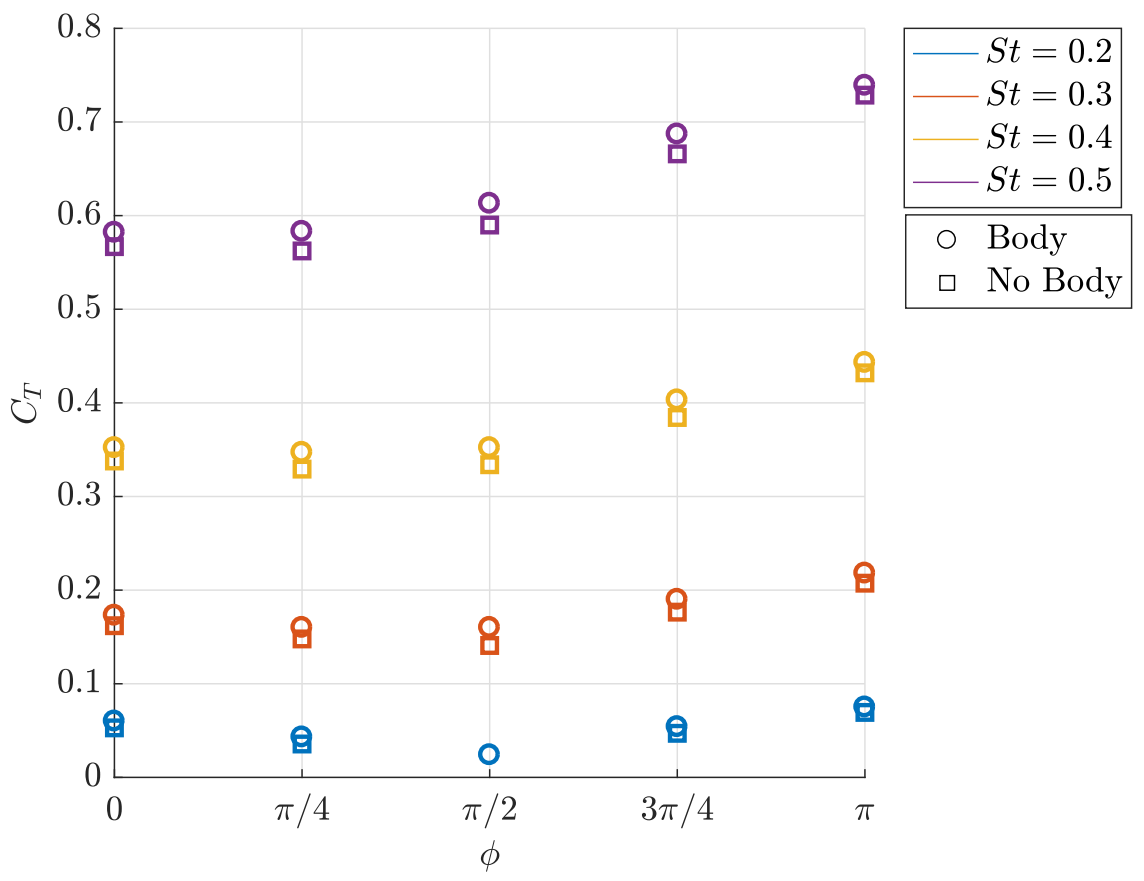

Figure 4.18: $C_{T}$ results for body and no body simulations. 


\section{Chapter 5}

\section{FUTURE WORK}

This work should serve as a starting point for more research into flapping foils and seal swimming at Cal Poly. There are several different research paths to build off this work. The fin shape is of significant importance in oscillatory mode swimming, and it has been shown that crescent shaped fins produces optimal results with regards to propulsive efficiency [31].

Changing the fin shape across constant Strouhal number and phase would provide insight into whether or not a certain shape is better at higher or lower values. Further research could investigate how adding heaving or flexing motions changes the hydrofoil performance behind a body. Alternatively, pitching fore flippers could be added to study the interactions between the upstream shed vortices and the downstream vortex generation. The foil spacing has also shown to be important but was kept constant in this work.

There are an endless number of different body shapes available to see how changing the disturbance affects propulsive performance. If the body is shaped more similarly to a seal, the point of maximum girth should be moved up towards the head in order to properly replicate the shoulder position.

If the long term goal is to realistically model a leopard seal swimming, then incorporating an undulating, flexible body with simultaneous pitching fore and hind flippers is the final iteration of this work. The hind flippers should have the ability to expand and contract while pitching.

Performing LES or DES on a similar model would improve the vortex shedding behavior. In addition to building off this CFD work, an experimental model could 
be designed and validated based off this data. If it were modular it would be very straightforward to study many combinations of body and foil shapes. This would also allow propulsive efficiency to be tested. 
Chapter 6

CONCLUSION

Unsteady Reynolds Averaged Navier-Stokes CFD was performed on tandem pitching hydrofoils behind a rigid prolate spheroid body for a range of Strouhal numbers (0.2$0.5)$ and phase differences $(0-\pi)$. Results of interest were the coefficient of thrust and the unsteady vorticity scene. The results were also compared to identical simulations without a upstream body to determine how the body affects thrust generation and the unsteady flow field.

Results indicate that adding an upstream prolate spheroid body does not significantly alter thrust results, though it does provide a small (nearly negligible) boost. Vorticity from the body is pulled downstream from the pitching foils, which interacts with the vortex generation. The vorticity pulled from the body does not affect the vorticity magnitude downstream.

The time averaged thrust increases with Strouhal number and is highest when the foils pitch out of phase with each other. When total thrust is broken into the trailing and leading results it is clear that the leading foil produces more thrust than the trailing foil, peaking at $21.7 \%$ more when $\phi=\pi / 2$ and $S t=0.5$. At $\phi=0$ and $\pi$ both foils generate equal amounts of thrust. These results show that there is a predictable Strouhal number trend, suggesting future research does not need to cover the entire Strouhal number range.

Increasing the phase difference by $\pi / 4$ advances the peak in instantaneous thrust generated by the leading foil by $f t=0.125$, so when $\phi=\pi / 2$ the leading foil peak aligns with the trailing foil trough, and when $\phi=\pi$ the second thrust peak of the leading foil lines up with the first peak from the trailing foil. 
This work raises several other questions. Further research into different body shapes is needed to confirm whether the shape or body length has any significant impact on the performance. More insight could be gained by changing the body shape to induce separation immediately upstream or in the foil vicinity. There is still much unknown regarding foil dynamics behind bodies. Modifying the fin shape and foil spacing would be the next logical steps from this study. Alternatively, improving the simulation quality by performing DES or LES would better model the vortex shedding.

Future work with an interest in leopard seal swimming should improve the model with more realistically shaped fins attached to a flexible body that can undulate. The fins should be able to contract and expand accordingly. Pitching fore flippers should also be introduced. 


\section{BIBLIOGRAPHY}

[1] Frank E Fish. Power output and propulsive efficiency of swimming bottlenose dolphins (Tursiops truncatus). Journal of Experimental Biology, 185(1):179-193, 1993.

[2] Anabela Maia, Alex P Sheltzer, and Eric D Tytell. Streamwise vortices destabilize swimming bluegill sunfish (Lepomis macrochirus). Journal of Experimental Biology, pages jeb-114363, 2015.

[3] Paul W Webb. The effect of solid and porous channel walls on steady swimming of steelhead trout Oncorhynchus mykiss. Journal of Experimental Biology, 178(1):97-108, 1993.

[4] Frank E Fish, Paul Legac, Terrie M Williams, and Timothy Wei. Measurement of hydrodynamic force generation by swimming dolphins using bubble dpiv. Journal of Experimental Biology, 217(2):252-260, 2014.

[5] Frank E Fish, S Innes, and K Ronald. Kinematics and estimated thrust production of swimming harp and ringed seals. Journal of Experimental Biology, 137(1):157-173, 1988.

[6] Eliot G Drucker and George V Lauder. A hydrodynamic analysis of fish swimming speed: wake structure and locomotor force in slow and fast labriform swimmers. Journal of Experimental Biology, 203(16):2379-2393, 2000.

[7] H Liu, Richard Wassersug, and Keiji Kawachi. The three-dimensional hydrodynamics of tadpole locomotion. Journal of Experimental Biology, 200(22):2807-2819, 1997. 
[8] Daniel Floryan, Tyler Van Buren, Clarence W Rowley, and Alexander J Smits. Scaling the propulsive performance of heaving and pitching foils. Journal of Fluid Mechanics, 822:386-397, 2017.

[9] Michael S Triantafyllou, Alexandra H Techet, and Franz S Hover. Review of experimental work in biomimetic foils. IEEE Journal of Oceanic Engineering, 29(3):585-594, 2004.

[10] Paul W Webb. Simple physical principles and vertebrate aquatic locomotion. American zoologist, 28(2):709-725, 1988.

[11] Frank E Fish. Influence of hydrodynamic-design and propulsive mode on mammalian swimming energetics. Australian Journal of Zoology, 42(1):79-101, 1994.

[12] Nigel Edwards, David Slip, Jeff Fung, Graham Doig, and Tracey L. Rogers. Re-evolution of a fore-limb propulsive swimming mode; the leopard seal a 'fast-lane- phocid.

[13] Voices in the Sea, 2018 (accessed December 11, 2018). http://cetus.ucsd.edu/voicesinthesea_org/index.html.

[14] Todd G Wetzel and Roger L Simpson. Unsteady flow over a 6: 1 prolate spheroid. Technical report, Virginia Polytechnic Inst and State Univ Blacksburg, 1996.

[15] Michael Goody, Roger Simpson, and Mark Engel. Mean velocity and pressure and velocity spectral measurements within a separated flow around a prolate spheroid at incidence. In 36th AIAA Aerospace Sciences Meeting and Exhibit, page 630, 1998. 
[16] Nathan Scott and Earl Duque. Unsteady reynolds-averaged navier-stokes predictions of the flow around a prolate spheroid. In 42nd AIAA Aerospace Sciences Meeting and Exhibit, page 55, 2004.

[17] Peter A Dewey, Daniel B Quinn, Birgitt M Boschitsch, and Alexander J Smits. Propulsive performance of unsteady tandem hydrofoils in a side-by-side configuration. Physics of Fluids, 26(4):041903, 2014.

[18] D Clarke, PA Brandner, and GJ Walker. Experimental and computational investigation of flow around a 3-1 prolate spheroid. WSEAS Transactions on Fluid Mechanics, 3(3):207-217, 2008.

[19] Nathan Scott and Earl Duque. Using detached eddy simulation and overset grids to predict flow around a 6: 1 prolate spheroid. In 43rd AIAA Aerospace Sciences Meeting and Exhibit, page 1362, 2005.

[20] Shin Hyung Rhee and Takanori Hino. Numerical simulation of unsteady turbulent flow around maneuvering prolate spheroid. AIAA journal, 40(10):2017-2026, 2002.

[21] Christer Fureby, Niklas Alin, Niklas Wikström, S Menon, N Svanstedt, and Leif Persson. Large eddy simulation of high-reynolds-number wall bounded flows. AIAA journal, 42(3):457-468, 2004.

[22] Daniel B Quinn, Keith W Moored, Peter A Dewey, and Alexander J Smits. Unsteady propulsion near a solid boundary. Journal of Fluid Mechanics, 742:152-170, 2014.

[23] Peter A Dewey, Birgitt M Boschitsch, Keith W Moored, Howard A Stone, and Alexander J Smits. Scaling laws for the thrust production of flexible pitching panels. Journal of Fluid Mechanics, 732:29-46, 2013. 
[24] George S Triantafyllou, MS Triantafyllou, and MA Grosenbaugh. Optimal thrust development in oscillating foils with application to fish propulsion. Journal of Fluids and Structures, 7(2):205-224, 1993.

[25] Birgitt M Boschitsch, Peter A Dewey, and Alexander J Smits. Propulsive performance of unsteady tandem hydrofoils in an in-line configuration. Physics of Fluids, 26(5):051901, 2014.

[26] R Gopalkrishnan, Michael S Triantafyllou, George S Triantafyllou, and D Barrett. Active vorticity control in a shear flow using a flapping foil. Journal of Fluid Mechanics, 274:1-21, 1994.

[27] Promode R Bandyopadhyay, John M Castano, William H Nedderman, and Martin J Donnelly. Experimental simulation of fish-inspired unsteady vortex dynamics on a rigid cylinder. Journal of Fluids Engineering, 122(2):219-238, 2000 .

[28] Hao Liu, R Wassersug, and Keiji Kawachi. A computational fluid dynamics study of tadpole swimming. Journal of Experimental Biology, 199(6):1245-1260, 1996.

[29] Patrick James Flanagan. Unsteady navier-stokes simulation of rainbow trout swimming hydrodynamics. 2004.

[30] Paul W Webb, Paul T Kostecki, and E Don Stevens. The effect of size and swimming speed on locomotor kinematics of rainbow trout. Journal of Experimental Biology, 109(1):77-95, 1984.

[31] Xinghua Chang, Laiping Zhang, and Xin He. Numerical study of the thunniform mode of fish swimming with different Reynolds number and caudal fin shape. Computers \& Fluids, 68:54-70, 2012. 
[32] Iman Borazjani and Mohsen Daghooghi. The fish tail motion forms an attached leading edge vortex. Proceedings of the Royal Society B: Biological Sciences, 280(1756):20122071, 2013.

[33] Geng Liu, Yan Ren, Haibo Dong, Otar Akanyeti, James C Liao, and George V Lauder. Computational analysis of vortex dynamics and performance enhancement due to body-fin and fin-fin interactions in fish-like locomotion. Journal of Fluid Mechanics, 829:65-88, 2017.

[34] Russell M Cummings, William H Mason, Scott A Morton, and David R McDaniel. Applied computational aerodynamics: A modern engineering approach, volume 53. Cambridge University Press, 2015.

[35] Philippe R Spalart and Steven R Allmaras. A one-equation turbulence model for aerodynamic flows. In 30th aerospace sciences meeting and exhibit, page 439, 1992.

[36] Tsan-Hsing Shih, William W Liou, Aamir Shabbir, Zhigang Yang, and Jiang Zhu. A new k- $\epsilon$ eddy viscosity model for high reynolds number turbulent flows. Computers \& Fluids, 24(3):227-238, 1995.

[37] Florian R Menter. Two-equation eddy-viscosity turbulence models for engineering applications. AIAA journal, 32(8):1598-1605, 1994.

[38] Patrick J Roache. Quantification of uncertainty in computational fluid dynamics. Annual Review of Fluid Mechanics, 29(1):123-160, 1997.

[39] Imran Akhtar, Rajat Mittal, George V Lauder, and Elliot Drucker. Hydrodynamics of a biologically inspired tandem flapping foil configuration. Theoretical and Computational Fluid Dynamics, 21(3):155-170, 2007.

[40] Siemens. STAR-CCM+ User Manual. Siemens. 
[41] Tracey L Rogers, Carolyn J Hogg, and A Irvine. Spatial movement of adult leopard seals (Hydrurga leptonyx) in Prydz Bay, eastern Antarctica. Polar Biology, 28(6):456-463, 2005.

[42] Thomas C Fu, A Shekarriz, Joseph Katz, and TT Huang. The flow structure in the lee of an inclined 6: 1 prolate spheroid. Journal of Fluid Mechanics, 269:79-106, 1994.

[43] James Lighthill. Hydromechanics of aquatic animal propulsion. Annual review of fluid mechanics, 1(1):413-446, 1969.

[44] Colin Southwell, Charles GM Paxton, David Borchers, Peter Boveng, Tracey Rogers, and K William. Uncommon or cryptic? challenges in estimating leopard seal abundance by conventional but state-of-the-art methods. Deep Sea Research Part I: Oceanographic Research Papers, 55(4):519-531, 2008.

[45] George S Constantinescu, Hugo Pasinato, You-Qin Wang, James R Forsythe, and Kyle D Squires. Numerical investigation of flow past a prolate spheroid. Journal of Fluids Engineering, 124(4):904-910, 2002. 